\title{
Preprocessing and Tightening Methods for Time-Indexed MIP Chemical Production Scheduling Models
}

\author{
Andres F. Merchan and Christos T. Maravelias* \\ Department of Chemical and Biological Engineering, University of Wisconsin-Madison \\ 1415 Engineering Dr., Madison WI, 53706, USA \\ E-mail: christos.maravelias@wisc.edu
}

\begin{abstract}
We propose a series of preprocessing algorithms for the generation of strong valid inequalities for time-indexed, discrete and continuous, mixed-integer programming scheduling models for problems in network production environments. Specifically, starting from time- and inventory-related instance data, the proposed algorithms use constraint propagation techniques to calculate parameters that are used to bound the number of times subsets of tasks can be executed in a feasible solution. We also extend some of the propagation ideas to generate three classes of new tightening constraints. The proposed methods result in tightening constraints expressed in terms of assignment binary variables $\left(X_{i j t}=1\right.$ if task $i$ is assigned to start on unit $j$ at time point $t$ ) which are present in all time-indexed MIP models, therefore they are applicable to all time-indexed models accounting for a wide range of processing features. Finally, the methods are shown to lead to up to two orders of magnitude reduction in computational time when optimal solutions are found and significantly improve optimality gap when a time limit is enforced.
\end{abstract}

Keywords: Constraint propagation, strong valid inequalities, preprocessing.

\section{INTRODUCTION}

The main focus of optimization-based scheduling in the process systems engineering (PSE) literature has been on the development of mixed-integer programming (MIP) models to address different classes of problems, rather than the development of solution methods (Mendez et al., 2006). Two main modeling approaches have been traditionally followed, batch-based and materialbased (Maravelias, 2012a; Harjunkoski et al., 2014). The former were developed to address problems in sequential environments, in which batch identity is preserved, meaning that once a batch enters the production sequence, it is neither mixed with other batches nor split into smaller amounts to be processed independently. The latter were developed to address problems in network environments where batch mixing and splitting as well as material recycling occur naturally.

Historically, the development of methods has closely followed this classification. Early work focused on problems in sequential environments, which are somewhat similar to discrete manufacturing problems, and relied on the assumption that the number of batches to be scheduled is known as well as a number of other assumptions regarding storage and utility constraints (Mauderli and Rippin, 1979; Reklaitis, 1978). Since then, multiple refinements have been proposed to increase the generality and efficiency of these models (Pinto and Grossmann, 1995; Pinto and Grossmann, 1998; Mendez et al., 2000; Gupta and Karimi, 2003; Castro and Grossmann, 2005). 
These models have also been extended to account for storage policies (Ku and Karimi, 1988; Mendez and Cerda, 2003) as well as utility constraints (Mendez et al., 2001). More recently, they were also extended to account for simultaneous batching and scheduling (Prasad and Maravelias, 2008; Sundaramoorthy and Maravelias, 2008b; Castro et al., 2008), including problems with storage (Sundaramoorthy and Maravelias, 2008a), utility (Sundaramoorthy et al., 2009), and other constraints (Baumann and Trautmann, 2013).

Methods for scheduling production in network environments were introduced in the early 1990s with the seminal work of Pantelides and coworkers (Kondili et al., 1993; Shah et al., 1993; Pantelides, 1994), which introduced the concepts of tasks and resources, as opposed to the single batch entity that characterized sequential environments. Despite the generality of these materialbased models, the computational capabilities at the time put a halt on the development of discretetime formulations, and researchers turned to smaller continuous-time models (Schilling and Pantelides, 1996; Zhang and Sargent, 1996; Ierapetritou and Floudas, 1998; Mockus and Reklaitis, 1999; Castro et al., 2001; Maravelias and Grossmann, 2003; Giannelos and Georgiadis, 2002; Sundaramoorthy and Karimi, 2005; Janak et al., 2006; Susarla et al., 2010; Gimenez et al., 2009b). However, continuous-time models are not as tight as their discrete-time counterparts, so the issue of computational efficiency remained unaddressed: industrial-scale problems are computationally intractable.

Recently, research in PSE has focused on the solvability of the chemical production scheduling models, including (1) the study of the structure of MIP modes (Pochet and Warichet, 2008; Maravelias and Papalamprou, 2009; Maravelias, 2012b); (2) decomposition algorithms (Bassett et al., 1996; Harjunkoski and Grossmann, 2002; Kelly and Zyngier, 2008); (3) tightening methods (Burkard and Hatzl, 2005; Janak and Floudas, 2008); (4) reformulations (Velez and Maravelias, 2013c; Velez and Maravelias, 2015); (5) algorithms that harness parallel computational resources (Subrahmanyam et al., 1996; Ferris et al., 2009; Velez and Maravelias, 2013a); and hybrid methods (Jain and Grossmann, 2001; Roe et al., 2005; Maravelias, 2006). Of particular interest for the algorithms discussed herein are the tightening methods of Maravelias and co-workers (Velez et al., 2013; Velez and Maravelias, 2013b; Merchan et al., 2013), where instance-specific demand information is used to generate tightening constraints for makespan or cost minimization problems subject to demand satisfaction constraints.

The goal of this paper is to develop methods for the generation of strong valid inequalities for profit or production maximization problems for which the previous methods cannot be used or are ineffective. Thus, rather than generating constraints that enforce lower bounds on the number of times tasks are executed (or the total production of materials and tasks), the methods discussed in the present paper lead to the generation of constraints upper bounding the number of batches. To this end, we use restrictions imposed by the availability of processing units and the initial inventories rather than restrictions imposed by demand.

The remaining of the paper is organized as follows. Section 2 presents background material, including the models we use to test our methods and several motivating examples to introduce the algorithms we propose. Section 3 contains the details of each of the algorithms that comprise our methods, with application to small-scale instances for illustration. In section 4 we extend our ideas from parameter calculation to constraint propagation. In section 5 we discuss how the computational implementation was carried out and section 6 presents the results of a comprehensive computational study. In Section 7, we apply our methods to an industrial example 
and we close in section 8, with concluding remarks and future directions. We use uppercase italic letters for variables, uppercase bold letters for sets, lowercase italic letters for indices, and lowercase Greek letters for parameters.

\section{BACKGROUND}

\subsection{Problem definition}

In its most general definition, a chemical production facility is a collection of units that transform a set of input materials into a set of added-value products. From the operating point of view, each unit executes a set of processing tasks that generate intermediate materials or final products by the chemical or physical transformation of raw or other intermediate materials. The fact that different tasks might share different resources requires the introduction of a time grid to keep track of the unit utilization and inventory levels for all the materials involved in the process. The concept of batch is then introduced to define a single execution of a task in a compatible unit. As mentioned in section 1 , we study production environments in which the identity of a single batch does not need to be tracked throughout the process because batch splitting and mixing are allowed (i.e. network environments). For these facilities, the chemical production scheduling problem can be defined as the problem of determining the number, size, assignment to units, and timing of individual batches for particular tasks.

Since the scheduling problem focuses on tasks rather than unit operations, a traditional process flow diagram representation of the plant is not useful, and an abstract representation of the production facility is required. Two commonly used representations are the State-Task Network, STN (Kondili et al., 1993) and the Resource-Task Network, RTN (Pantelides, 1994). The former represents the production plant as a network in which the nodes define tasks and materials, the arcs represent streams for the flow of materials between tasks, and the units are implicitly mapped to compatible tasks. The latter explicitly includes the processing units as nodes in the network, and unify them with the materials under a common family of resources that can be used by tasks at different moments. Our methods are independent of the abstract representation and can be applied to either STN- or RTN-based optimization models. The present work uses the STN representation, for which the following sets are required to define the structure of the plant:

$\mathbf{I}=\{i: i$ is a task $\}$

$\mathbf{J}=\{j: j$ is a processing unit $\}$

$\mathbf{J}_{i}=\{j \in \mathbf{J}:$ unit $j$ can process task $i\}$

$\mathbf{I}_{j}=\{i \in \mathbf{I}:$ task $i$ can be processed in unit $j\}$

$\mathbf{K}=\{k: k$ is a material $\}$

$\mathbf{I}_{k}^{+} / \mathbf{I}_{k}^{-}=\{i \in \mathbf{I}:$ task $i$ produces/consumes material $k\}$

$\mathbf{K}_{i}^{+} / \mathbf{K}_{i}^{-}=\{k \in \mathbf{K}$ : material $k$ is produced/consumed by task $i\}$

The main assumptions we make for our STN-based formulations are (a) no preemption is allowed and (b) the problem data are deterministic. The derivation of our methods does not depend on most of the commonly found features such as additional resources (utilities, labor), changeovers, shared storage vessels and variable processing times. Therefore, our methods are applicable to problems that include these features. In particular, they can be directly applied to problems with additional resources, while minor changes are required to include formulations of changeovers, shared storage and variable processing time. 
A specific instance of a process network is defined using the following parameters:

$\beta_{j}^{\max } / \beta_{j}^{\min }: \quad$ Maximum/minimum batch size for unit $j \in \mathbf{J}$

$\bar{\tau}_{i j}: \quad$ Processing time for task $i \in \mathbf{I}$ in unit $j \in \mathbf{J}_{i}$

$\gamma_{k}: \quad$ Storage capacity for material $k \in \mathbf{K}$

$\xi_{k 0}: \quad$ Initial inventory of material $k \in \mathbf{K}$

$\rho_{i k}$ : $\quad$ Conversion coefficient for material $k \in \mathbf{K}$ produced $\left(\rho_{i k}>0\right)$ or consumed $\left(\rho_{i k}<0\right)$ by task $i \in \mathbf{I}$

The final element required for the abstract definition of the scheduling problem in network environments is the definition of a feasible schedule through three main requirements: (1) a unit can perform at most one task at a given time (i.e. unit utilization); (2) the batch sizes satisfy the processing unit capacities; and (3) material inventory satisfy nonnegativity and storage vessel capacity constraints (Maravelias, 2012a). In section 2.2 we discuss how these requirements are mathematically modeled using MIP formulations.

\subsection{Mathematical models}

The development of a mathematical model for scheduling requires that we make a decision about the type of time representation to be used. In discrete-time models, the scheduling horizon, $\eta$, is divided into a set of equal time periods of length (step size) $\delta$ (such that $\eta / \delta \in \mathbb{Z}$ ); we also use a set of points $\mathbf{T}=\{t \in \mathbb{Z}: 0 \leq t \leq \eta / \delta\}$. In continuous-time formulations, the horizon is divided into $N$ time intervals of unknown length that in turn define the set of time points $\mathbf{N}=\{n \in \mathbb{Z}: 0 \leq n \leq N\}$. Interval $n$ runs between points $n-1$ and $n$, and since the position of the time point is not known $a$ priori, it has to be included as a decision variable.

The decision variables that are common to both time representations are:

$X_{i j t} / X_{i j n}: \quad$ Binary. It is equal to one if task $i \in \mathbf{I}$ starts in unit $j \in \mathbf{J}_{i}$ at time $t \in \mathbf{T} / n \in \mathbf{N}$

$B S_{i j t} / B S_{i j n}: \quad$ Continuous nonnegative. Batch size of task $i \in \mathbf{I}$ that starts in unit $j \in \mathbf{J}_{i}$ at time $t \in \mathbf{T} / n \in \mathbf{N}$

$S_{k t} / S_{k n}: \quad$ Continuous nonnegative. Inventory level of material $k \in \mathbf{K}$ at time point $t \in \mathbf{T} / n \in \mathbf{N}$ with $S_{k 0}=\xi_{k 0}$

Most continuous-time models require the following additional variables:

$T_{n}: \quad$ Continuous nonnegative. Actual value of time point $n \in \mathbf{N}$

$Y_{i j n}: \quad$ Binary. It is equal to one if task $i \in \mathbf{I}$ finishes in unit $j \in \mathbf{J}_{i}$ at time $n \in \mathbf{N}$

$B F_{i j n} / B P_{i j n}$ : Continuous nonnegative. Batch size of task $i \in \mathbf{I}$ that starts/continues to be processed in unit $j \in \mathbf{J}_{i}$ at time $n \in \mathbf{N}$

The main difference between discrete- and continuous-time models appears when the unit utilization constraint is enforced. In discrete-time models, it is typically modeled using the singlemachine clique constraint (Nemhauser and Wolsey, 1988), while continuous-time formulations require multiple types of constraints to map the tasks onto the variable time grid. The batch size and material balance constraints remain essentially the same for both types of time representations. In the following subsections, we present the models that we use to test our methods. However, we note that the methods are applicable to all time-indexed material-based MIP models.

\subsubsection{SP\&S model}


The discrete-time model we consider was developed by Shah et al. (Shah et al., 1993) and in this work it is referred to as the SP\&S model. It consists of single equations to model each of the three scheduling constraints discussed in section 2.1. Equation 1 is the clique constraint that represents unit utilization, equation 2 restricts the batch size, and equation 3 expresses the material balance and enforces the storage vessel capacity.

$\sum_{i \in \mathbf{I}_{j}} \sum_{t^{\prime}=t-\tau_{i j}+1}^{t} X_{i j t^{\prime}} \leq 1 \quad \forall j \in \mathbf{J}, t \in \mathbf{T}$

$\beta_{j}^{\text {min }} X_{i j t} \leq B S_{i j t} \leq \beta_{j}^{\max } X_{i j t} \quad \forall i \in \mathbf{I}, j \in \mathbf{J}_{i}, t \in \mathbf{T}$

$S_{k t}=S_{k(t-1)}+\sum_{i \in \mathbf{I}_{k}^{+}} \sum_{j \in \mathbf{I}_{i}} \rho_{i k} B S_{i j\left(t-\tau_{i j}\right)}+\sum_{i \in \mathbf{I}_{k}^{-}} \sum_{j \in \mathbf{I}_{i}} \rho_{i k} B S_{i j t}+\xi_{k t} \leq \gamma_{k} \quad \forall k \in \mathbf{K}, t \in \mathbf{T}$

Note that in equations 1 and 3, the processing time is expressed in terms of the number of time points, by using $\tau_{i j}=\left\lceil\bar{\tau}_{i j} / \delta\right\rceil$. Equation 3 also includes the parameter $\xi_{k t}$ that represents the delivery $\left(\xi_{k t}>0\right)$ or demand $\left(\xi_{k t}<0\right)$ of material $k \in \mathbf{K}$ at time $t \in \mathbf{T}$.

\subsubsection{S\&K model}

The first continuous-time model we use was originally developed by Sundaramoorthy and Karimi (Sundaramoorthy and Karimi, 2005) and we refer to it as the S\&K model. It relies on four separate balances to model the three scheduling constraints: resource and processing time balance to enforce unit utilization, material residing in process units to satisfy batch sizes, and material inventory in storage vessels to enforce material balances and storage capacities. They also modified the definition of the set of tasks, by introducing an idle task to occupy a time slot when no task is assigned to a particular unit, although no batch sizing variables are explicitly assigned to such a task. As a result we redefine $\mathbf{I}=\{i: i$ is a task $\} \cup\{i d l e\}$. Full details of model $\mathrm{S} \& \mathrm{~K}$ are given in Appendix A.

\subsubsection{GH\&M model}

The second continuous-time model we study was proposed by Gimenez et al. (Gimenez et al., 2009a). It addresses most of the features that appear in chemical production scheduling, but we only use those parts of the model that make it comparable to the S\&K model. Specifically, we do not consider explicit modeling of material transfer and shared vessel utilization. This formulation is based on the current state of a processing unit: execution, storage or idle. Time balances are used to model unit utilization, while batch size and material balance constraints are analogous to those in S\&K model. We refer to this formulation as the GH\&M model and present the complete list of equations in Appendix A.

\subsection{Motivating examples}

In this section we introduce the basic concepts that will be used to develop the new methods we propose by means of small-scale illustrations. Each instance highlights the benefit of specific algorithms that are then fully developed in section 3.

\subsubsection{Time availability and inventory restrictions}

The first concept we use is the time window to define the effective time a task could actually be carried out within the scheduling horizon. Although in principle the entire horizon is available for 
the execution of the tasks, limitations in the inventory of input and output materials of a given task shorten the time available for its execution. To calculate the time window of a specific task we use the concepts of earliest start time (EST) and latest finish time (LFT), which have been used before in the scheduling literature (He and Hui, 2006; Castro et al., 2009). The novelty of our approach, however, is that we consider not only processing times, but also required amounts of materials associated with a task to calculate EST. For convenience, we define the shortest tail (ST) of a task as the difference between the horizon and the LFT. Thus, tasks that exclusively produce final products could finish at the very end of the scheduling horizon (i.e. a zero shortest tail), whereas the rest of the tasks should finish earlier so the intermediates they produce have enough time to be converted into final products. The time window for a specific task is then calculated by subtracting the EST and ST values for that task from the total horizon.

Consider the network depicted in Figure 1. We present next the necessary conceptual steps to calculate the time window for a particular task. A formal discussion and generalization is presented in section 3.1. Let us calculate the EST for task T3, which is nonzero since the initial inventory for material S3 is zero. Note that at least $25 \mathrm{~kg}$ of S3 are required to start running T3. The earliest start time for T1 is zero, since S1 has enough inventory. We use data on processing time and unit capacity to calculate maximum amounts that could be produced at different times by T1. Figure 2 shows these calculations and establishes that the EST for T3 in U3 is 4 hours, which is the time required to produce at least $25 \mathrm{~kg}$ of S3. Note that if only data for processing time were considered, an incorrect value of 2 hours would be calculated. The shortest tail for T3 is calculated by considering the executions that lead to production of final product S6, and not those that only increase the inventory of intermediate material S5. Since S5 has to be consumed by task T4 in unit $\mathrm{U} 4$, for which the processing time is 2 hours, we conclude that, for a horizon $\eta=12 h$, the LFT for task T3 is 10 hours and therefore its ST is 2 hours. Consequently, the effective time window for task T3 is 6 hours $(=\eta-E S T-S T=12-4-2)$. Figure 3 presents the results for time windows of all tasks depicted in Figure 1, with and without considering inventories of intermediates.

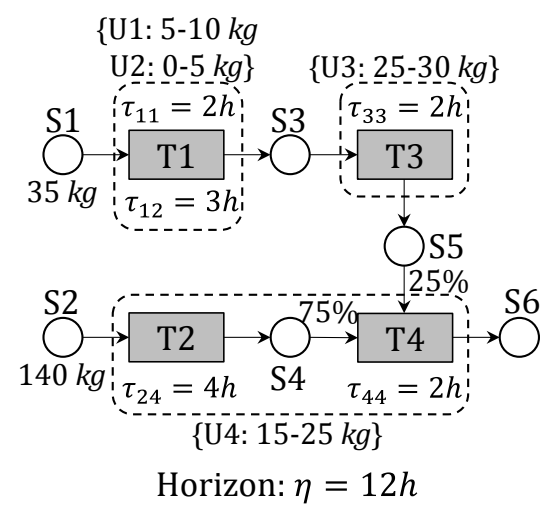

Figure 1. STN representation for the first illustrative example.

Unit-task compatibility and data for processing times, unit capacities, initial inventory, and conversion coefficients are provided. 


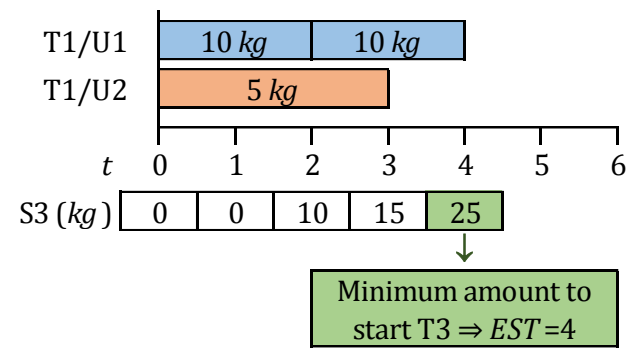

Figure 2. EST calculation for task T3.

The earliest start time for task T3 of the illustrative example is $4 h$, which is the time it takes to produce $25 \mathrm{~kg}$ of material S3 to satisfy its minimum batch size.

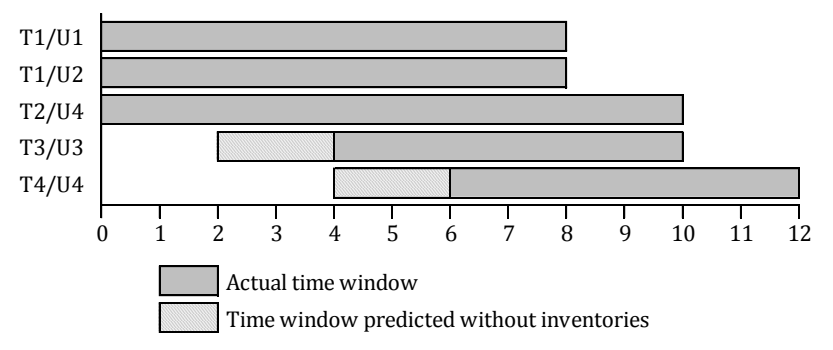

Figure 3. Time windows for all tasks in the first illustrative example.

If inventory of intermediates is not taken into account, longer and incorrect time windows are predicted.

Once the time windows have been calculated for each task, we propagate the information regarding initial inventories for raw materials and intermediates following the direction of the material flow (i.e. forward propagation). This proagation allows the calculation of the maximum cumulative production of each material. Once this value has been determined for all input materials of a task, the maximum cumulative amount that the task can process based on material inventories is calculated. This value can then be compared with the maximum amount the task could process within its time window, based on the maximum capacity of the units in which it can be processed. The minimum of the last two values defines the maximum cumulative production for the specific task.

To illustrate these concepts we consider task T3 of the network in Figure 1. We fix the initial inventory of raw material S1 to $35 \mathrm{~kg}$ and begin the forward propagation. Based on its time window, T1 could process $50 \mathrm{~kg}$ of $\mathrm{S} 1$ (maximum capacity $\left|\frac{\text { timewindow }}{\text { processing time }}\right|$; from U1: $40=10$. $[8 / 2]$, and from $U 2: 10=5 \cdot[8 / 3]$ ) but there are only $35 \mathrm{~kg}$ of S1 available, so T1 is limited by inventory and its maximum cumulative production is $35 \mathrm{~kg}$. This value can in turn be propagated to material S3, to define its maximum cumulative production. Based on the time window for T3, a maximum of $90 \mathrm{~kg}(=30 \cdot[6 / 2])$ can be processed, so we conclude that T3 is also inventorylimited and its maximum cumulative production is $35 \mathrm{~kg}$.

An additional refinement can be made by analyzing the feasible batch sizes derived from the unit capacities. Task T3 is processed in unit U3, whose capacity is $[25,30] \mathrm{kg}$. This means that one batch of T3 in U3 can produce a maximum of $30 \mathrm{~kg}$ and two batches produce at least $50 \mathrm{~kg}$. There is no way to produce exactly $35 \mathrm{~kg}$, so the maximum cumulative production of T3 is adjusted to $30 \mathrm{~kg}$.

When we include information on available time and maximum cumulative production in the MIP formulations, the corresponding LP relaxations are improved. For instance, if the production of product S6 is maximized, the LP relaxations of the three models we consider (SP\&S, S\&K, GH\&M) 
predict $60 \mathrm{~kg}$ of $\mathrm{S} 6$. However, when time and inventory restrictions are included in the model, the LP relaxations improve for models SP\&S and GH\&M. The former predicts a value of $50 \mathrm{~kg}$, whereas the latter calculates a value of $57.14 \mathrm{~kg}$. Note that the integer solution obtained when integrality is enforced is $50 \mathrm{~kg}$, which coincides with the LP relaxation for model SP\&S. Although in more complex networks the improved results do not match the integer solution, the introduction of these methods greatly enhances the efficiency of the solution procedures as shown in section 6 .

\subsubsection{Task dependence and feasible number of batches}

Given a specific network, it is possible to identify groups of dependent tasks for which their number of batches are correlated. Being able to identify these groups and generate equations to represent the dependence between the number of batches of different tasks within a group is one of the key components of our methods.

Consider the process network shown in Figure 4. We can identify three different groups of tasks based on different types of interactions that render their number of batches dependent. First, tasks $\mathrm{T} 1$ and $\mathrm{T} 2$ can be grouped together because they share unit U1. Although we can identify separate time windows for each task, the dependence appears when the unique time window for unit U1 is considered. If we assume that S1 and S2 have large inventories at the beginning of the horizon, then the earliest start time for both $\mathrm{T} 1$ and $\mathrm{T} 2$ is zero. From the processing time data we calculate shortest tail values of $3 h$ and $2 h$ for T1 and T2 respectively. Consequently, the time window for T1 is $7 h$ and for T2 is $8 h$ and since the inventory of S1 and S2 is large enough, both tasks are timelimited. The maximum number of batches of $\mathrm{T} 1$ and $\mathrm{T} 2$ that can be processed independently $\left.\left(\mid \frac{\text { time window }}{\text { processing time }}\right]\right)$ are $N_{T 1}^{\max }=3(=[7 / 2\rfloor)$ and $N_{T 2}^{\max }=4(=[8 / 2])$. However, since they share a common unit with a time window of $8 h$ (using minimum values of earliest start time and shortest tail), only some combinations of number of batches are feasible, as shown in Figure 5.

Second, tasks T4 and T5 form a new group because they share input material S4. Let us assume that the maximum cumulative amount of S4 is $100 \mathrm{~kg}$. Individual estimates for the maximum number of batches of T4 and T5 $\left(\left\lfloor\frac{\text { Cumulative input }}{\text { Conversion-Min Capacity }}\right]\right)$ result in $N_{T 4}^{\max }=2(=[100 /(1 \cdot 40)\rfloor)$ and $N_{T 5}^{\max }=$ $3(=\lfloor 100 /(1 \cdot 30)])$. Once more, the shared resource results in reduced feasible combinations of batches, as presented in Figure 6.

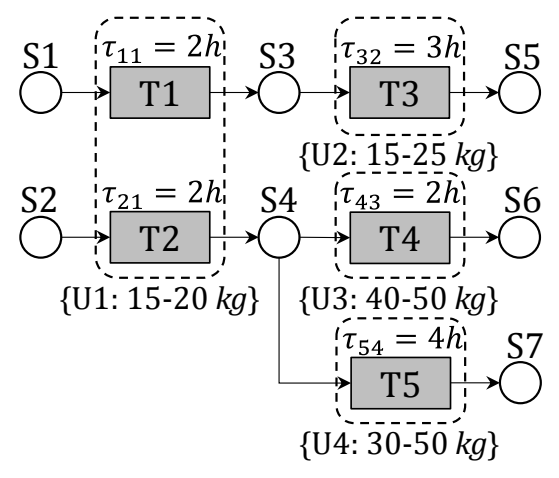

Horizon: $\eta=10 h$

Figure 4. STN representation for second illustrative example.

Unit-task compatibility and data for processing times and unit capacities are provided.

Third, tasks T3 and T4 constitute a less intuitive group, since they consume materials produced by the same unit. Intermediates S3 and S4 are both produced by unit U1, which induces a dependence 
between T3 and T4. The simple forward-propagation algorithm would suggest that the number of batches of T3 and T4 are not related, but since the cumulative production of S3 and S4 is affected by the production in unit $U 1$, we have to propagate the available information on $U 1$ to determine if the required amounts of input materials constitute a feasible production scenario. In this case, there is not a single closed-form constraint that can be used to determine the feasible set of points. As we discuss in section 3.2, a more sophisticated procedure is required to identify such a set. Figure 7 shows the feasible combinations of batches obtained when this dependence is taken into account.

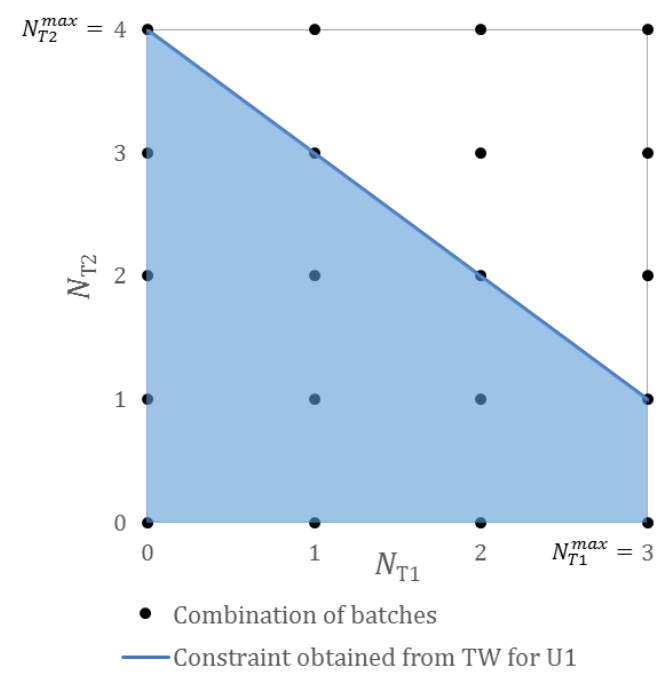

Figure 5. Group of dependent tasks that share a common processing unit.

If tasks T1 and T2 are considered independent then $\left(N_{T 1}, N_{T 2}\right) \in[0,3] \times[0,4]$. Since they share unit U1 we can write $2 N_{T 1}+2 N_{T 2} \leq 8$ and $N_{T 1} \leq 3$, based on the time window for the unit. Here $N_{T 1}=\sum_{t} X_{T 1, U 1, t}$ and $N_{T 2}=\sum_{t} X_{T 2, U 1, t}$

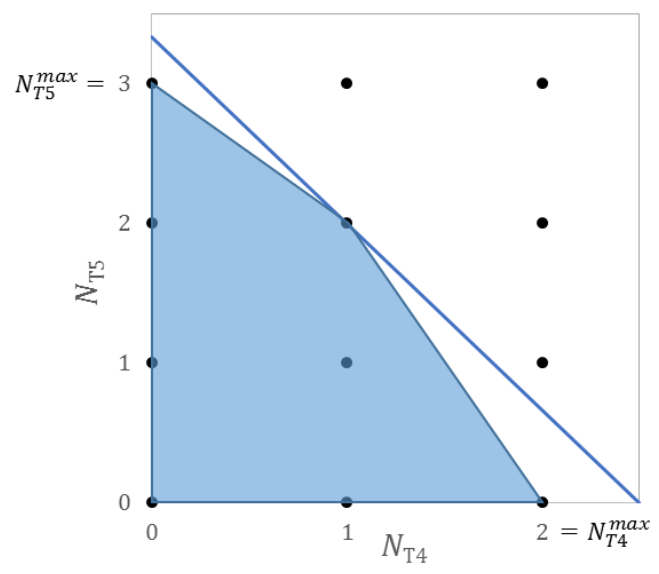

- Combination of batches

Constraint obtained from S4 prodution

Figure 6. Group of dependent tasks that share a common input material.

If tasks T4 and T5 are considered independent then $\left(N_{T 4}, N_{T 5}\right) \in[0,2] \times[0,3]$. Since they share input material S4 we can first write $40 N_{T 4}+30 N_{T 5} \leq 100$ based on cumulative production of the material. Moreover, we can determine the convex hull of the feasible integer points to obtain $N_{T 4}+N_{T 5} \leq 3$ and $2 N_{T 4}+N_{T 5} \leq 4$. Here $N_{T 4}=\sum_{t} X_{T 4, U 3, t}$ and $N_{T 5}=\sum_{t} X_{T 5, U 4, t}$. 
The analysis of the three types of groups described above leads to the generation of constraints describing the convex hull of feasible combinations of number of batches for the tasks within the group. The tightening effect is shown in Figures 5 through 7, in which some portions of the search space of the LP-relaxation are removed. For more complex instances, in which combinations of time- and inventory-limited tasks are present, this tightening can be greatly beneficial as discussed in section 6.

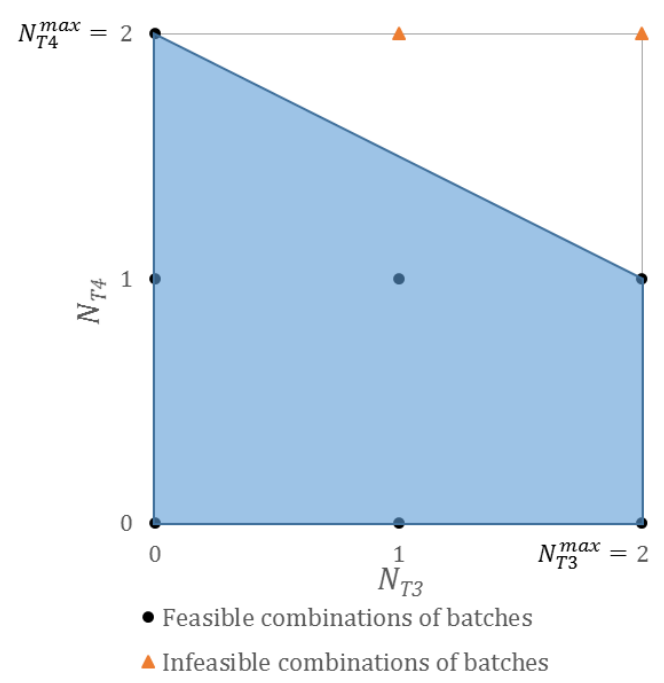

Figure 7. Group of dependent tasks that consume materials produced by a common unit.

If tasks T3 and T4 are considered independent then $\left(N_{T 3}, N_{T 4}\right) \in[0,2] \times[0,2]$. Since they consume materials S3 and S4 both produced by unit U1, there is an implicit dependence, that renders the combinations $\left(N_{T 3}, N_{T 4}\right) \in\{(1,2),(2,2)\}$ infeasible. The convex hull of the feasible integer points can be used to obtain $N_{T 3}+2 N_{T 4} \leq 4$ and $N_{T 3} \leq 2$. Here $N_{T 3}=\sum_{t} X_{T 3, U 2, t}$ and $N_{T 4}=\sum_{t} X_{T 4, U 3, t}$.

\section{PROPOSED METHODS}

The methods we propose are based on preprocessing instance data (processing time, initial inventory, conversion coefficients, and unit capacities) to obtain parameters that are used to generate tightening constraints. We discuss a sequence of algorithms that identify the initial grouping of tasks and calculate parameters that are propagated following the flow of materials in the network. These parameters are then used to write tightening constraints. In order to execute the proposed algorithms, we need to define new sets and parameters as follows.

$d \in \mathbf{D} \quad$ Groups of dependent tasks

$\mathbf{I}_{d} \quad$ Tasks that belong to group $d$

$\mathbf{J}_{d} \quad$ Units that belong to group $d$

$h \in \mathbf{H}_{d} \quad$ Equations derived from group $d$

$\varepsilon_{i j} \quad$ Earliest start time for task $i \in \mathbf{I}$ in unit $j \in \mathbf{J}_{i}$

$\sigma_{i j} \quad$ Shortest tail for task $i \in \mathbf{I}$ in unit $j \in \mathbf{J}_{i}$

$\theta_{i j} \quad$ Time window for task $i \in \mathbf{I}$ in unit $j \in \mathbf{J}_{i}$

$\mu_{i} \quad$ Maximum cumulative production of task $i \in \mathbf{I}$ within the given horizon

$\omega_{k} \quad$ Maximum cumulative production of material $k \in \mathbf{K}$ within the given horizon

$\varphi_{i d h} \quad$ Coefficient for task $i \in \mathbf{I}_{d}$ in equation $h \in \mathbf{H}_{d}$

$\varphi_{d h} \quad$ Right-hand-side coefficient in equation $h \in \mathbf{H}_{d}$ 
The definition of time window is direct, based on the discussion presented in section 2.3.1:

$\theta_{i j}=\eta-\varepsilon_{i j}-\sigma_{i j} \quad \forall i \in \mathbf{I}, \forall j \in \mathbf{J}_{i}$

We define four basic algorithms: (1) time window (TW) algorithm, (2) forward-propagation (FP) algorithm, (3) group identification (GI) algorithm, and (4) feasible region's convex hull (FR) algorithm. Each of these algorithms defines and calculates some of the parameters that we just introduced. The flowchart in Figure 8 shows the sequence of calculations and how the different algorithms interact with each other. Subsequent subsections explain each algorithm in detail.

\subsection{Time Window (TW) Algorithm}

The first algorithm is divided into two steps; for every task in the network one step calculates the earliest start time (TW_EST) and the other computes the shortest tail (TW_ST). The TW_EST step calculates $\varepsilon_{i j}$ by finding the time value for which the maximum amount of a specific input material, $k \in \mathbf{K}_{i}^{-}$, that can be produced up to that time exceeds the minimum amount required to execute one batch of task $i$. This is illustrated for task T3 of the motivating example presented in section 2.3.1 using Figure 9. The stair-shaped line in Figure 9a represents the production profile for material S3 if the tasks producing it used all available units at their maximum capacities. The dotted line is the minimum amount of material required for a single batch of task T3. The time at which these two lines intersect represents the earliest start time for T3 in unit U3.

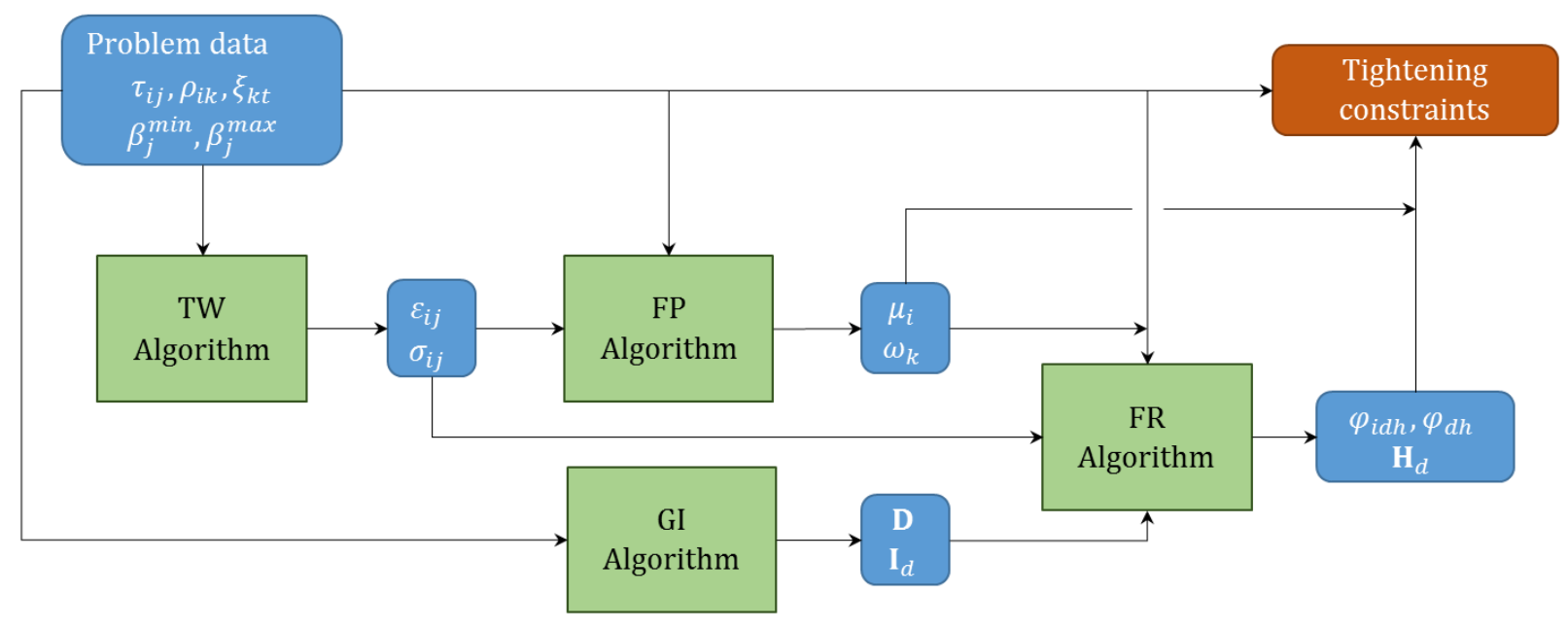

Figure 8. Flowchart for the proposed methods.

Flow of information, results of each algorithm and interactions among them are shown. 


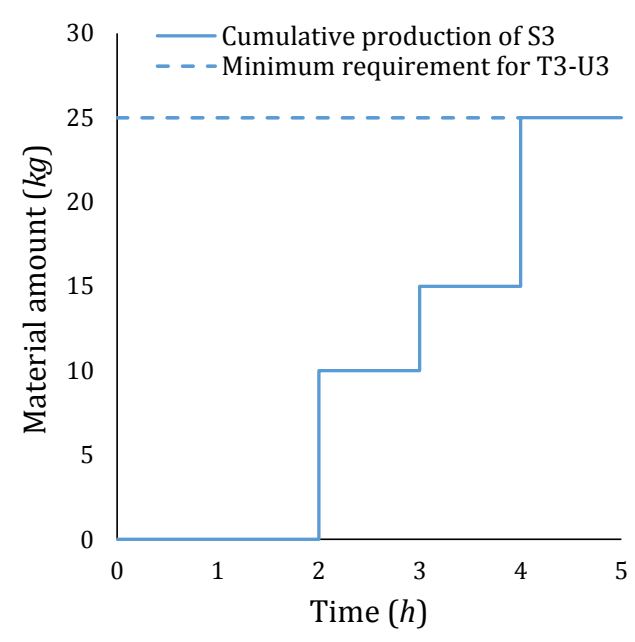

(a)

$$
\begin{aligned}
& i=T 3, j=U 3, \mathbf{K}_{T 3}^{-}=S 3 \\
& \mathbf{I}_{S 3}^{+}=\{T 1\}, \mathbf{K}_{T 1}^{-}=\{S 1\}, \rho_{T 1, S 3}=1, \rho_{T 1, S 1}=-1 \\
& \bar{t} \quad \psi_{S 1, \bar{t}}^{E} \quad \psi_{S 3, \bar{t}}^{E} \quad \chi_{T 1, U 1, \bar{t}}^{E} \chi_{T 1, U 2, \bar{t}}^{E} \quad \phi_{T 1, \bar{t}}^{E} \quad \chi_{T 3, U 3, \bar{t}}^{E} \\
& \begin{array}{lllllll}
0 & 35 & 0 & 10 & 5 & 15 & 0
\end{array} \\
& \begin{array}{lllllll}
1 & 35 & 0 & 10 & 5 & 15 & 0
\end{array} \\
& \begin{array}{lllllll}
2 & 35 & 10 & 20 & 5 & 25 & 0
\end{array} \\
& \begin{array}{lllllll}
3 & 35 & 15 & 20 & 10 & 30 & 0
\end{array} \\
& \begin{array}{lllllll}
4 & 35 & 25 & 30 & 10 & 40 & 25
\end{array} \\
& \Rightarrow \varepsilon_{T 3, U 3}=\min \left\{\bar{t}: \chi_{T 3, U 3, \bar{t}}^{E}>0\right\}=4
\end{aligned}
$$

(b)

Figure 9. (a) Cumulative production profile for material S3 and minimum input material requirement for task T3 in unit U3, for the illustrative example in Figure 1. (b) Calculation steps for the TW_EST step.

The TW_EST step uses the following parameters in order to keep track of the two separate quantities:

$\psi_{k \bar{t}}^{E}$

Maximum amount of material $k \in \mathbf{K}$ available at time $\bar{t}$

$\chi_{i j \bar{t}}^{E}$

Cumulative production for task $i \in \mathbf{I}$ in unit $j \in \mathbf{J}_{i}$ starting at or before time $\bar{t}$

$\phi_{i \bar{t}}^{E}$

Cumulative production for task $i \in \mathbf{I}$ in any unit starting at or before time $\bar{t}$

The TW_EST step is based on a predefined time grid $\bar{t} \in \mathbf{T}$ over which it loops to determine the earliest time a task can start. In discrete-time models this grid coincides with the discretization introduced by the step size $\delta$, i.e. $\overline{\mathbf{T}}=\mathbf{T}$. In continuous-time formulations, an auxiliary grid is defined using two steps: (1) all the processing times are rounded to a fixed number of decimal places, and (2) a step size equal to the greatest common factor of the processing times is introduced. Equation 5 defines $\psi_{k \bar{t}}^{E}$ as the total supply of material $k$ plus the maximum amount that can be produced up to time $\bar{t}$. The second term is calculated as the minimum between two quantities: (1) the amount of material $k$ that can be produced in all units that process tasks producing $k$ up to time $\bar{t}$, and (2) the amount of material $k$ produced by all tasks producing $k$ up to time $\bar{t}$.

$\psi_{k \bar{t}}^{E}=\sum_{0 \leq \bar{t}^{\prime} \leq \bar{t}} \xi_{k \bar{t}^{\prime}}+\sum_{i \in \mathbf{I}_{k}^{+}}\left[\rho_{i k} \min \left\{\sum_{j \in J_{i}} \chi_{i j\left(\bar{t}-\tau_{i j}\right)}^{E}, \phi_{i\left(\bar{t}-\min _{j \in J_{i}} \tau_{i j}\right)}^{E}\right\}\right]$

Equation 6 defines $\chi_{i j \bar{t}}^{E}$, which is initialized based on the amount of input material $k$ available for task $i$ (first term), but cannot increase by more than $\beta_{j}^{\text {max }}$ every $\tau_{i j}$ time periods (second term). Equation 6 also expresses that if $\chi_{i j \bar{t}}^{E}$ is less than the minimum unit capacity, $\beta_{j}^{\text {min }}$, then no material can be processed by task $i$ in unit $j$ and therefore $\chi_{i j \bar{t}}^{E}=0$.

$\chi_{i j \bar{t}}^{E}=\min \left\{\min _{k \in \mathbf{K}_{i}^{-}}\left(-\psi_{k \bar{t}}^{E} / \rho_{i k}\right), \chi_{i j\left(\bar{t}-\tau_{i j}\right)}^{E}+\beta_{j}^{\max }\right\}$; if $\chi_{i j \bar{t}}^{E}<\beta_{j}^{\text {min }}$ then set $\chi_{i j \bar{t}}^{E} \leftarrow 0$ 
Next, equation 7 provides the value of $\phi_{i \bar{t}}^{E}$ based on the total amount of input material available. This value is at most the sum of the amounts that can be produced in all units that process task $i$.

$\phi_{i \bar{t}}^{E}=\min \left\{\min _{k \in \mathbf{K}_{i}^{-}}\left\{-\psi_{k \bar{t}}^{E} / \rho_{i k}\right\}, \sum_{j \in \mathbf{J}_{i}} \chi_{i j \bar{t}}^{E}\right\}$

Finally, the earliest start time is the first time point for which $\chi_{i j \bar{t}}^{E}$ is nonzero as expressed through equation 8.

$\varepsilon_{i j}=\min \left\{\bar{t}: \chi_{i j \bar{t}}^{E}>0\right\}$

Figure 10 presents the outline of the TW_EST step. We use the set $\mathbf{T}^{E x p}$ to define the set of time points that need to be explored, according to the time grid used. Figure 11 shows the procedure of the TW_EST step when applied to the motivating example introduced in section 2.3.1.

The TW_ST step is simpler than the TW_EST step and uses a modification of the shortest path algorithm (Dijkstra, 1959) as described by Velez and Maravelias (Velez and Maravelias, 2013b). It uses the following sets and parameters:

Sets

$\mathbf{K}^{F}=\{k \in \mathbf{K}: k$ is a raw material $\}$

$\mathbf{K}^{P}=\{k \in \mathbf{K}: k$ is a final product $\}$

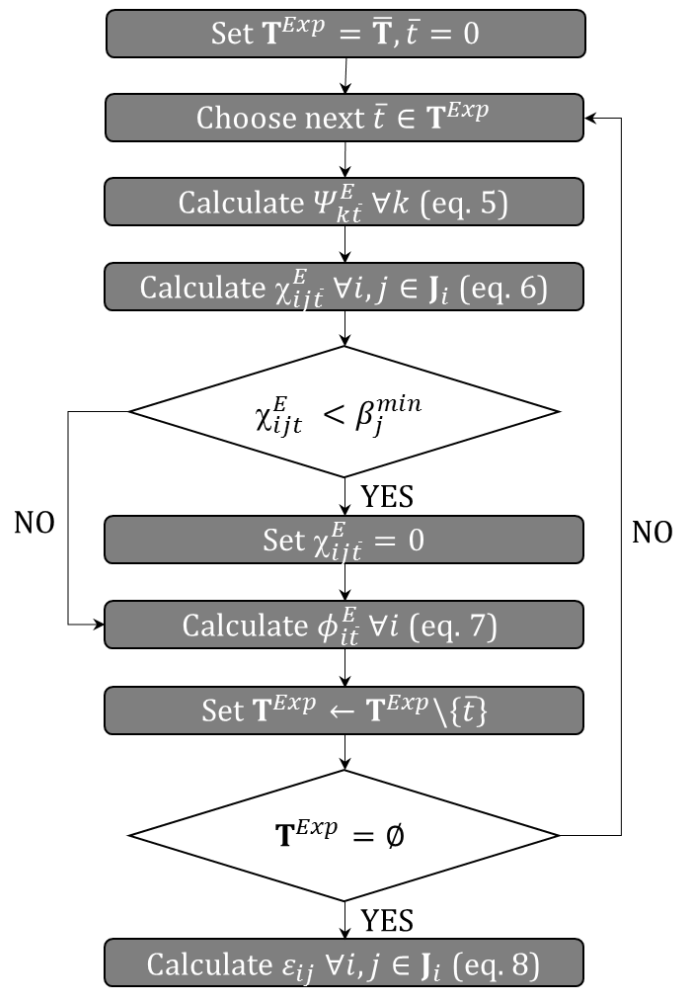

Figure 10. Flowchart for the TW_EST step in TW algorithm.

Parameters

$\phi_{k}^{S} \quad$ Minimum time required to process material $k \in \mathbf{K}$ so that it leads to production of final products. 

have enough time to be transformed into final products.

The TW_ST step starts by assigning large values of $\phi_{k}^{S}$ to raw and intermediate materials and zero values to materials in $\mathbf{K}^{P}$. It also initializes $\lambda_{i j}^{S}$ with large values. The set $\mathbf{K}^{E x p}$ is used to keep track of the materials that need to be evaluated. Equation 9 is used to calculate $\lambda_{i j}^{S}$ in terms of $\phi_{k}^{S}$, and equation 10 to calculate $\phi_{k}^{S}$ based on tasks that consume material $k$. Finally, equation 11 is used to calculate the shortest tail $\sigma_{i j}$. Figure 12 presents the calculations of the TW_ST step.

$\lambda_{i j}^{S}=\min _{k \in \mathbf{K}_{i}^{+}} \phi_{k}^{S} \quad \forall i \in \mathbf{I}_{k}^{+}, j \in \mathbf{J}_{i}$

$\phi_{k}^{S}=\min _{i \in \mathbf{I}_{j}}\left\{\phi_{k}^{S}, \lambda_{i j}^{S}+\bar{\tau}_{i j}\right\} \quad \forall k \in \mathbf{K}_{i}^{-}$

$\sigma_{i j}=\eta-\lambda_{i j}^{S} \quad \forall i \in \mathbf{I}_{k}^{+}, j \in \mathbf{J}_{i}$

Figure 13 shows the application of the TW_ST step to the motivating example in section 2.3.1.

As mentioned in section 2.3.1, by extension it is possible to calculate the earliest start time and shortest tail of individual units, as well as the corresponding time windows as shown in equations 12-14. These parameters are important in developing the FR algorithm in section 3.4 and additional tightening constraints in section 3.5 .

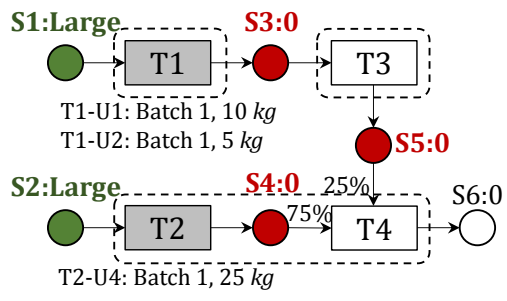

(a) $\bar{t}=\mathbf{0 h}$

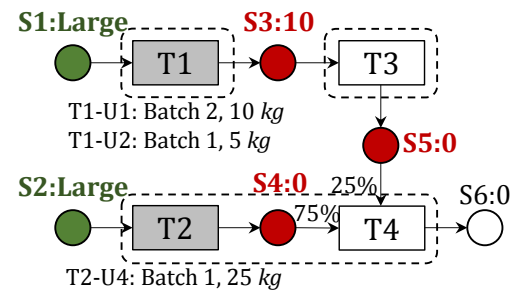

(b) $\bar{t}=2 h$

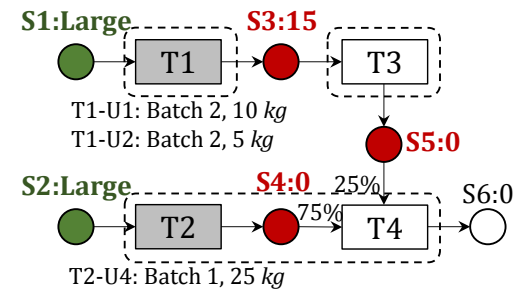

(c) $\bar{t}=3 h$

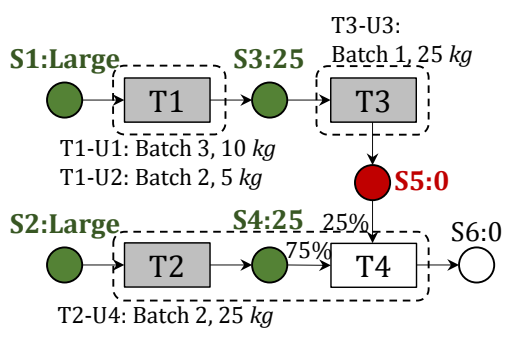

(d) $\bar{t}=4 \mathrm{~h}$

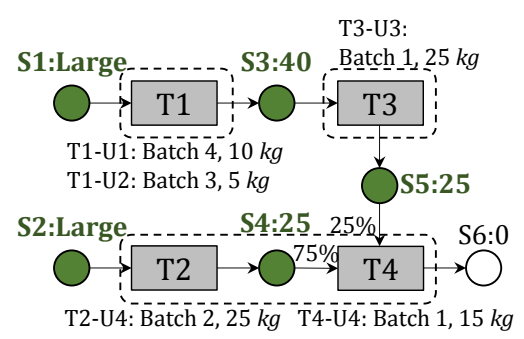

(e) $\bar{t}=6 h$

Figure 11. Calculation procedure for the TW_EST step for the process network in Figure 1.

Evolution of cumulative production at times when batches of different tasks start/finish. A green material indicates its cumulative amount is enough to start tasks that consume it. A red material indicates its cumulative amount is below the minimum required to start tasks consuming it. A gray task indicates it is ready to process its first batch. A white task is still waiting for its input materials to reach minimum levels to start. The value of $\varepsilon_{i j}$ corresponds to the first $\bar{t}$ where the task is gray. Minimum requirements for tasks are T3: $25 \mathrm{~kg}$ of S3, T4: $11.25 \mathrm{~kg}$ of S4 and $3.75 \mathrm{~kg}$ of S5. From Figure (a) we obtain $\varepsilon_{T 1, U 1}=\varepsilon_{T 1, U 2}=\varepsilon_{T 2, U 4}=0$. From Figures (d) an (e) respectively we get $\varepsilon_{T 3, U 3}=4$ and $\varepsilon_{T 4, U 4}=6$. 


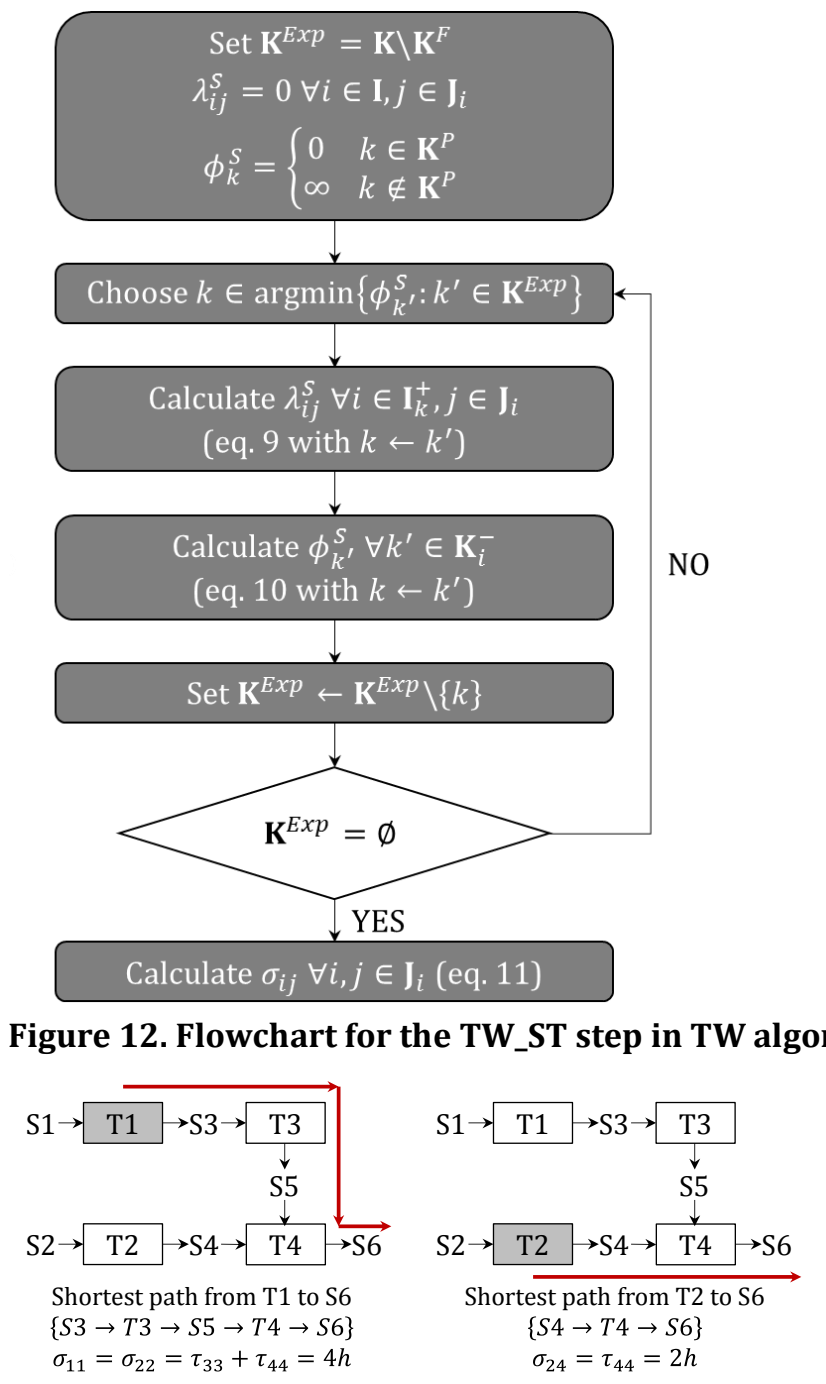

\section{Set $\mathbf{K}^{E x p}=\mathbf{K} \backslash \mathbf{K}^{F}$ \\ $\lambda_{i j}^{S}=0 \forall i \in \mathbf{I}, j \in \mathbf{J}_{i}$ \\ $\phi_{k}^{S}= \begin{cases}0 & k \in \mathbf{K}^{P} \\ \infty & k \notin \mathbf{K}^{P}\end{cases}$}

Figure 12. Flowchart for the TW_ST step in TW algorithm.
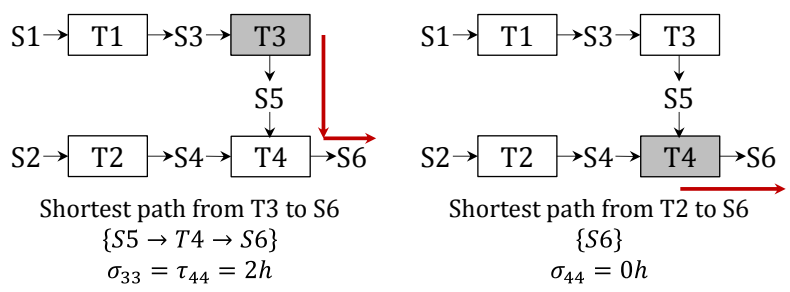

Figure 13. Calculation procedure for the TW_EST step.

$\varepsilon_{j}=\min _{i \in \mathbf{I}_{j}} \varepsilon_{i j} \quad \forall j \in \mathbf{J}$

$\sigma_{j}=\min _{i \in \mathbf{I}_{j}} \sigma_{i j} \quad \forall j \in \mathbf{J}$

$\theta_{j}=\eta-\varepsilon_{j}-\sigma_{j} \quad \forall j \in \mathbf{J}$

\subsection{Forward-Propagation (FP) Algorithm}

The maximum cumulative production for task $i, \mu_{i}$, and material $k, \omega_{k}$, are calculated through an algorithm that propagates the inventory and time availability information in the direction of the material flow within the network. This forward-propagation algorithm generalizes the ideas presented in section 2.3.1 for the illustrative example and is based on the comparison between values predicted for the maximum amount that a task can process when time and inventory availability are considered separately. Based on time, this maximum amount can be calculated once the time window for the task is known using equation 15. 
$\mu_{i}^{T W}=\sum_{j \in \mathbf{J}_{i}} \beta_{j}^{\max }\left\lfloor\frac{\theta_{i j}}{\tau_{i j}}\right\rfloor \quad \forall i \in \mathbf{I}$

To consider inventory availability we have to solve the linear program defined in equation 16 , in which we maximize the amount produced by task $i$, represented by the variable $Q_{i}$. The constraints in this LP enforce that (i) the amount produced of each material plus any initial inventory is greater than the amount consumed, and that (ii) for each material the total consumption by downstream tasks does not exceed its maximum feasible production within the given horizon. Note that the LP in equation 16 implicitly uses the structure of the network, so it is valid for networks with or without recycled materials.

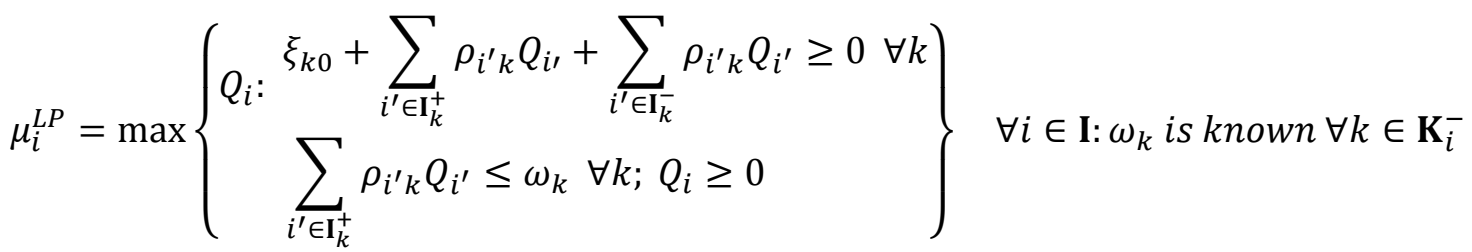

Equation 17 sets the maximum production of the task to the minimum predicted using time window and inventory restrictions. Finally, equation 18 allows the propagation of information to the materials produced by tasks whose $\mu_{i}$ is known.

$\mu_{i}=\min \left\{\mu_{i}^{T W}, \mu_{i}^{L P}\right\} \quad \forall j \in \mathbf{J}$

$\omega_{k}=\xi_{k 0}+\sum_{i \in \mathbf{I}_{k}^{+}} \rho_{i k} \mu_{i}^{1} \quad \forall k \in \mathbf{K}: \mu_{i}$ is known $\forall i \in \mathbf{I}_{k}^{+}$

Equation 18 introduces a new parameter, $\mu_{i}^{1}$, based on the ideas discussed by Velez et al. (Velez et al., 2013). A correction in the original value of $\mu_{i}$ predicted through equation 17 is required, since the capacities of the units might prevent this exact amount to be processed. Further details on this correction procedure are presented in Appendix B.

Figure 14 summarizes the complete forward-propagation algorithm. The sets $\mathbf{I}^{E x p}$ and $\mathbf{K}^{\operatorname{Exp}}$ respectively contain the tasks and materials that have been explored. Figure 15 presents the results of the application of the FP algorithm to the motivating example introduced in Figure 1.

\subsection{Group Identification (GI) Algorithm}

After time availability has been calculated for each task in the network through the time window $\theta_{i j}$, and the forward-propagation has been executed to predict maximum feasible productions for both tasks $\left(\mu_{i}\right)$ and materials $\left(\omega_{k}\right)$, a new procedure is introduced to define the feasible combinations of the number of batches in subgroups of dependent tasks; i.e., tasks that are interrelated and whose production is limited by one another. There are three different types of groups: (1) Type I are tasks that share a processing unit; (2)Type II are tasks that share an upstream material; and (3) Type III are tasks that consume materials produced by a common upstream unit. The first two types are simpler to analyze, since both the time window for the common unit and the maximum production for the shared material bound the maximum combined amount the tasks within the group can process. The third type also defines upper bounds on the combined processing amounts; however, the identification of a group and the feasible combination 
of batches requires the propagation of Type I constraints. The output of this algorithm is the set of dependent groups $d \in \mathbf{D}$.

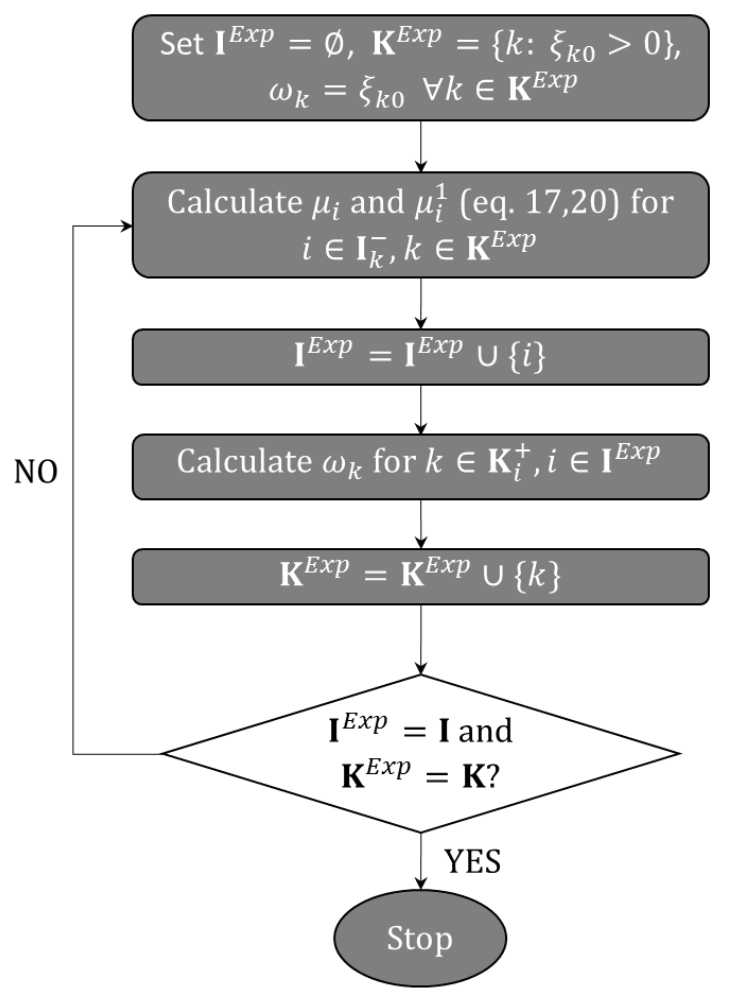

Figure 14. Flowchart for the FP Algorithm.

Calculates the maximum feasible production amounts for each task, $\mu_{i}$, and material, $\omega_{k}$, within the network.

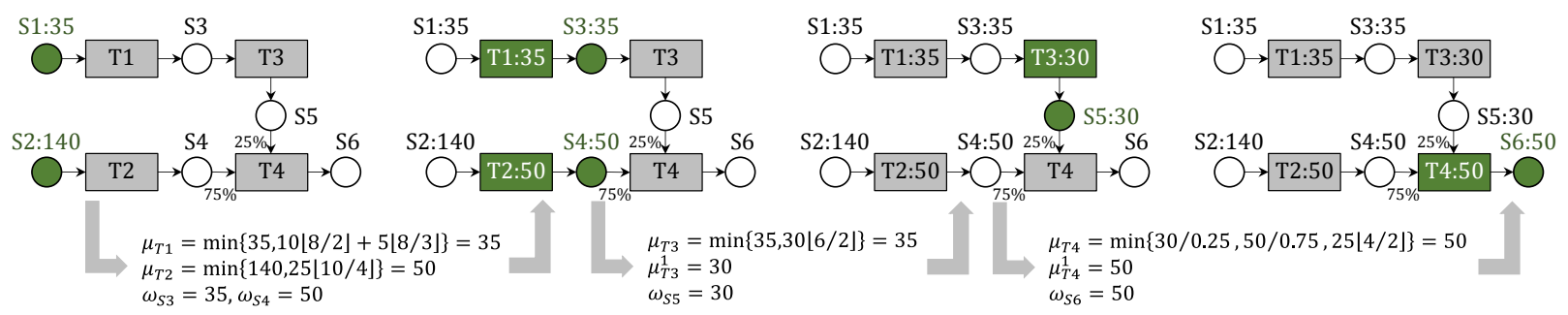

Figure 15. Calculation steps and results for FP algorithm applied to network in Figure 1.

Materials and tasks in green indicate their parameters, $\omega_{k}$ and $\mu_{i}$ respectively, are calculated in a specific step.

Figure 16 shows the basic structure of the GI Algorithm, where the following additional sets are defined:

$\mathbf{J}_{k}^{+}\left(\mathbf{J}_{k}^{-}\right)=\left\{j \in \mathbf{J}_{i}: i \in \mathbf{I}_{k}^{+}\left(\mathbf{I}_{k}^{-}\right)\right\} \quad$ Units that produce (consume) material $k$

$\mathbf{K}_{j}^{+}\left(\mathbf{K}_{j}^{-}\right)=\left\{k \in \mathbf{I}_{k}^{+}\left(\mathbf{I}_{k}^{-}\right): i \in \mathbf{I}_{j}\right\} \quad$ Materials produced (consumed) by task $j$

As an illustration, we revisit the motivating example presented in section 2.3.2 and focus on the Type II group. We follow the algorithm in Figure 16 to confirm the qualitative analysis we performed in section 2.3.2 where we identified that tasks T4 and T5 are dependent because they consume a common material. We select $i=T 4$, so the relevant sets are $\mathbf{K}_{T 4}^{-}=\{S 4\}$ and $\mathbf{I}_{S 4}^{-} \backslash\{T 4\}=\{T 5\}$. Without loss of generality, let us assume that the counting index is set at $d \leftarrow 1$. 
Then, the new group is defined as $\mathbf{I}_{1}=\{T 4, T 5\}$, the counting index is increased by one (i.e. $d \leftarrow 2$ ), and $\mathbf{I}_{2}$ is initialized as an empty set. The algorithm continues until all tasks have been visited and grouped.

\subsection{Feasible Region (FR) Algorithm}

For each of the groups identified through the GI algorithm and indexed by $d$, the maximum number of batches of each task in the group, $\zeta_{i d}^{\max }$, is calculated using equation 19 that considers restrictions in both available time and inventory. The former involves exploring all the time windows in compatible units for task $i$, whereas the latter finds the most restrictive input material for task $i$ over all the compatible units.

$\zeta_{i d}^{\max }=\min \left\{\sum_{j \in \mathbf{J}_{i}}\left\lfloor\frac{\theta_{i j}}{\tau_{i j}}\right\rfloor, \max _{j \in \mathbf{J}_{i}}\left(\min _{k \in \mathbf{K}_{i}^{-}}\left\lfloor\frac{\omega_{k}}{\rho_{i k} \beta_{j}^{\min }}\right\rfloor\right)\right\} \quad \forall d \in \mathbf{D}, i \in \mathbf{I}_{d}$

Subsequently, the set of points $P$ for the feasible combinations of the number of batches of each task in the group is determined, depending on the type of dependence. For Type I, the time representation is important since it is based on the time window for a unit. Equations 20 and 21 are respectively used in continuous-time and discrete-time formulations to limit the possible combinations of batches for tasks within the group.

$\sum_{i \in \mathbf{I}_{j}} \sum_{t \in \mathbf{T}} \bar{\tau}_{i j} X_{i j t} \leq \theta_{j}$

$\sum_{i \in I_{j}} \sum_{t} \tau_{i j} X_{i j t} \leq\left\lfloor\theta_{j} / \delta\right\rfloor \quad \forall j \in \mathbf{J}$

For Type II groups, equation 22 is used to determine the set $P$, based on the available amount of the shared material. 


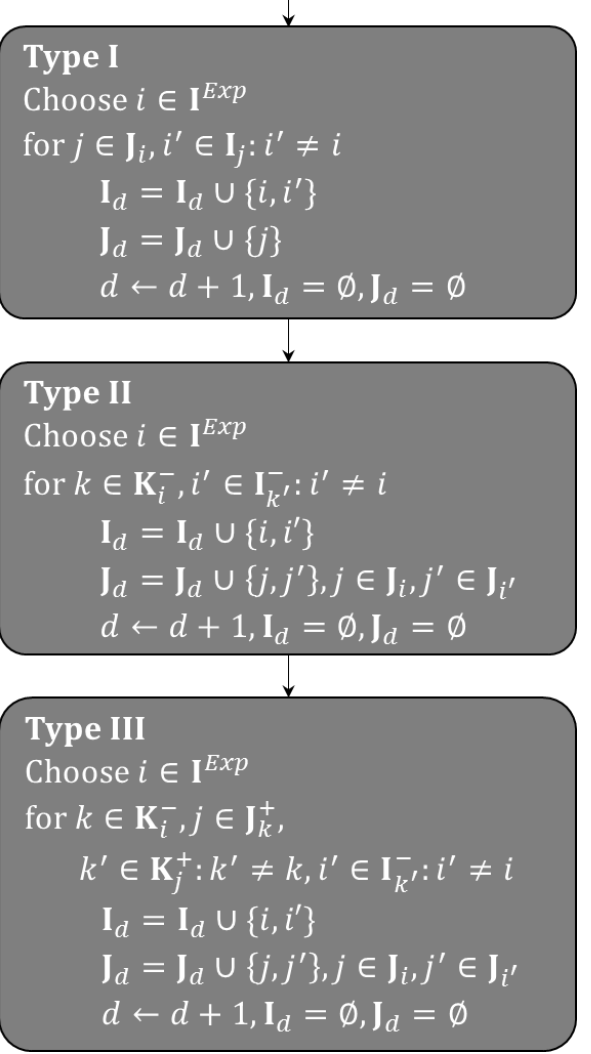

Figure 16. Flowchart for the GI algorithm.

It is used to find the groups of interdependent tasks. Type I corresponds to tasks sharing a unit, Type II appears when tasks share a common input material, and Type III groups tasks that use materials produced by the same unit.

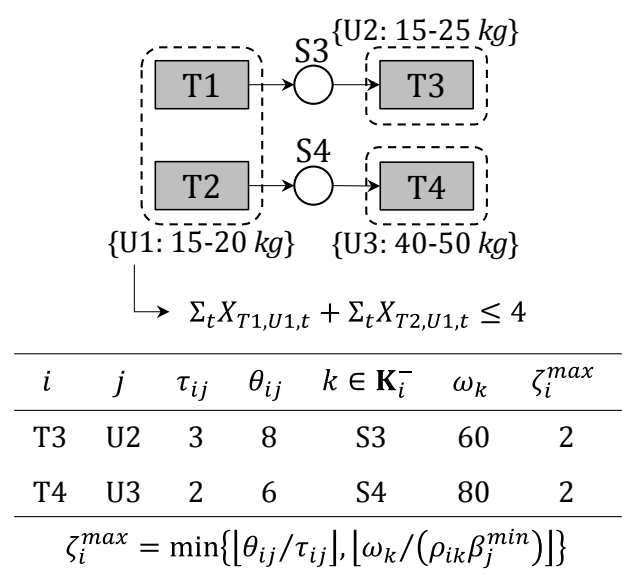

Figure 17. Analysis of Type III group.

Application to example in Figure 4 (partial STN is shown)

$\sum_{i \in \mathbf{I}_{k}^{-}} \sum_{j \in \mathbf{J}_{i}} \sum_{t \in \mathbf{T}} \beta_{j}^{\text {min }} \rho_{i k} X_{i j t} \leq \omega_{k}$

Tasks in Type III groups consume materials produced by a common upstream unit. This processing unit necessarily defines a Type I group, for which a set of constraints can be found based on 
equations 20 or 21 . These constraints can be then forward-propagated to determine feasible combination of batches for tasks in the Type III group. As an illustration, let us revisit the example presented in section 2.3.2. Figure 17 presents the portion of the network that corresponds to the Type III group with the relevant parameters associated to its tasks and the Type I constraint derived from unit $\mathrm{U} 1$.

Using the Type I constraint in terms of the assignment variable and the unit capacity constraint from the original model (equations 2, A8, and A25 for models SP\&S, S\&K, GH\&M respectively), we can derive an equation in terms of batch sizes for tasks in unit U1: $\hat{B}_{T 1}+\widehat{B}_{T 2} \leq 80$, where $\hat{B}_{i}=\sum_{t} B S_{i, U 1, t}$ is the cumulative production of task $i$. If we define $W_{k}$ to be the cumulative production of material $k \in \mathbf{K}_{i}^{+}, i \in \mathbf{I}_{U 1}$, we can upper-bound it using $\hat{B}_{i}: W_{S 3} \leq \rho_{T 1, S 3} \hat{B}_{T 1} ; W_{S 4} \leq$ $\rho_{T 2, S 4} \hat{B}_{T 2}$. Therefore we can evaluate if a combination of batches of tasks T3 and T4 is feasible, by calculating the required values of S3 and S4, and evaluating feasibility using the constraints we just generated. For instance, in Figure 7 we show that the combination of one batch of T3 and two batches of T4 is infeasible. This combination requires $W_{S 3} \geq 1 \cdot \rho_{T 3, S 3} \beta_{U 2}^{\min } ; W_{S 4} \geq 2 \cdot \rho_{T 4, S 4} \beta_{U 3}^{\min }$. But we can directly see that $\left\{\left(\hat{B}_{T 1}, \hat{B}_{T 2}, W_{S 3}, W_{S 4}\right): \hat{B}_{T 1}+\hat{B}_{T 2} \leq 80,30 \leq W_{S 3} \leq \hat{B}_{T 1} ; 80 \leq W_{S 4} \leq\right.$ $\left.\hat{B}_{T 2}\right\}=\varnothing$ and therefore the combination is infeasible.

We now generalize this procedure by formally defining two new variables: (1) $\hat{B}_{i j}=\sum_{t} B S_{i j t}$, the cumulative production of task $i$ in unit $j \in \mathbf{J}_{i}$ and (2) $W_{k}$, the cumulative production of material $k \in \mathbf{K}_{i}^{+}, i \in \mathbf{I}_{j}$ is a Type I group. We also define the required amount of input materials for $v_{i^{\prime} j^{\prime}}$ batches of task $i^{\prime} \in \mathbf{I}_{k}^{-}$in unit $j^{\prime} \in \mathbf{J}_{i^{\prime}}$ as $\omega_{k}^{R}=v_{i^{\prime} j^{\prime}} \rho_{i^{\prime} k} \beta_{j^{\prime}}^{\min }$. Then, the LP feasibility problem in equation 23 is solved for each combination of batches up to the maximum value for each task.

$$
\left\{\begin{aligned}
\left(\hat{B}_{i j}, W_{k}\right): & \sum_{i \in \mathbf{I}_{j}} \varphi_{i j h} \hat{B}_{i j} \leq \varphi_{j h} \beta_{j}^{\text {max }}: \mathbf{I}_{j} \text { is a Type I group, } h \in \mathbf{H}_{j} \\
& \omega_{k}^{R} \leq W_{k} \leq \sum_{i \in \mathbf{I}_{k}^{+}} \sum_{j \in \mathbf{J}_{i}} \rho_{i k} \hat{B}_{i j} \forall k
\end{aligned}\right\}
$$

Finally, once the set of points has been identified for each type of group, its convex hull is obtained by using the qhull algorithm (Barber et al., 1996). The inequalities that describe $\operatorname{conv}(P)$ can directly be added to the discrete- or continuous-time formulations to further tighten the original models. Figure 18 summarizes the basic steps in the FR algorithm, where the set $\mathbf{D}^{\operatorname{Exp}}$ contain the groups of dependent tasks that still need to be analyzed.

Let us consider again the example presented in section 2.3.2, and focus on the Type II group previously identified by the GI algorithm, i.e. $\{T 4, T 5\}$. Equation 24 in this case becomes $\sum_{i \in \mathbf{I}_{S 4}^{-}} \sum_{j \in \mathbf{J}_{i}} \beta_{j}^{\text {min }}\left|\rho_{i, S 4}\right| \sum_{t \in \mathbf{T}} X_{i j t} \leq \omega_{S 4}$, the relevant sets are $\mathbf{I}_{S 4}^{-}=\{T 4, T 5\}, \mathbf{J}_{T 4}=\{U 3\}, \mathbf{J}_{T 5}=\{U 4\}$ and the important parameters $\rho_{T 4, S 4}=\rho_{T 5, S 4}=-1, \beta_{U 3}^{\min }=40, \beta_{U 4}^{\min }=30, \omega_{S 4}=100$. After simplification, we obtain the constraint $4 \sum_{t \in \mathbf{T}} X_{T 4, U 3, t}+3 \sum_{t \in \mathbf{T}} X_{T 5, U 4, t} \leq 10$, which is depicted in Figure 6 . The set of possible combinations of batches of T4 and T5 is then:

$$
P=\{(0,0),(1,0),(2,0),(0,1),(1,1),(0,2),(1,2),(0,3)\}
$$

And after applying the qhull algorithm we obtain: 


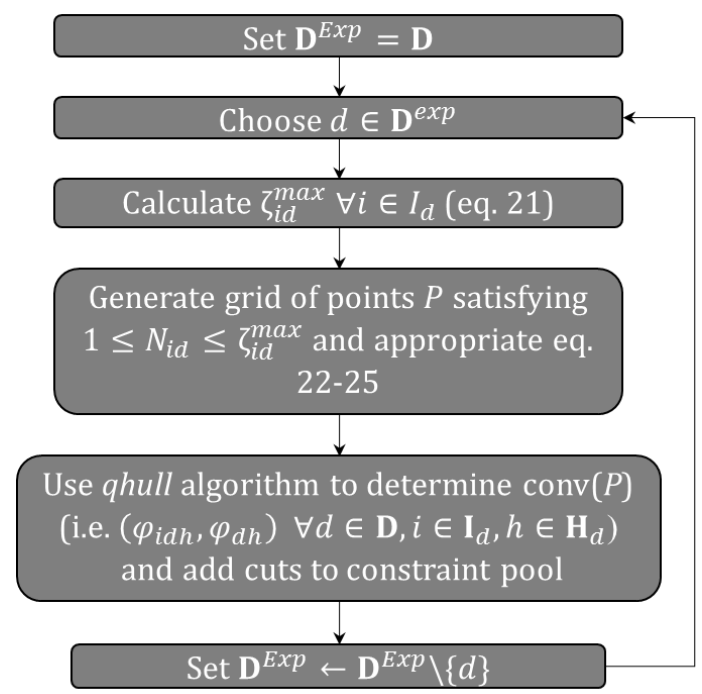

Figure 18. Flowchart for the FR algorithm.

This algorithm provides the coefficients for the linear combinations defining feasible combinations of batches within a given dependent group.

$$
\operatorname{conv}(P)=\left\{\begin{aligned}
\left(\sum_{t \in \mathbf{T}} X_{T 4, U 3, t}, \sum_{t \in \mathbf{T}} X_{T 5, U 4, t}\right): & \sum_{t \in \mathbf{T}} X_{T 4, U 3, t} \geq 0, \sum_{t \in \mathbf{T}} X_{T 5, U 4, t} \geq 0, \\
& \sum_{t \in \mathbf{T}} X_{T 4, U 3, t}+\sum_{t \in \mathbf{T}} X_{T 5, U 4, t} \leq 3, \\
& 2 \sum_{t \in \mathbf{T}} X_{T 4, U 3, t}+\sum_{t \in \mathbf{T}} X_{T 5, U 4, t} \leq 4
\end{aligned}\right\}
$$

This set is represented by the shadowed area in Figure 6.

\subsection{Constraints}

In this section we explain how the parameters calculated through the four algorithms are used to define different sets of tightening constraints that can be added to any discrete- or continuous-time formulation. For continuous-time models we assume that the processing time is fixed and does not depend on the batch size.

In continuous-time models, equation 20 can be directly used to bound the linear combination that defines the total time spent by a task in a single unit by using the time window defined through the TW Algorithm. Equation 24 is the equivalent constraint for a specific task.

$\sum_{i \in I_{j}} \sum_{t} \bar{\tau}_{i j} X_{i j t} \leq \theta_{j} \quad \forall j \in \mathbf{J}$

$\sum_{t} \bar{\tau}_{i j} X_{i j t} \leq \theta_{i j} \quad \forall i, j \in \boldsymbol{J}_{i}$

The corresponding equations for discrete-time formulations are given by 21 and 25 .

$$
\sum_{i \in \boldsymbol{I}_{j}} \sum_{t} \tau_{i j} X_{i j t} \leq\left\lfloor\theta_{j} / \delta\right\rfloor \quad \forall j \in \mathbf{J}
$$


$\sum_{t} \tau_{i j} X_{i j t} \leq\left\lfloor\theta_{i j} / \delta\right\rfloor \quad \forall i, j \in \boldsymbol{J}_{i}$

The total cumulative production for a given task can be bounded above by $\mu_{i}^{1}$ calculated in the FP Algorithm, regardless of time representation.

$\sum_{j \in \mathbf{J}_{i}} \sum_{t} B S_{i j t} \leq \mu_{i}^{1} \quad \forall i \in \mathbf{J}$

Finally, the parameters calculated using the FR Algorithm are used to constrain a linear combination of batches of dependent tasks belonging to a group:

$\sum_{i \in \mathbf{I}_{d}, j \in \mathbf{J}_{i}, t} \varphi_{i d h} X_{i j t} \leq \varphi_{d h} \quad \forall d, h \in \mathbf{H}_{d}$

\section{EXTENSION TO VARIABLE EST AND ST}

A generalization of the concepts of earliest start time and shortest time to end of the horizon is proposed in order to obtain even tighter formulations, when the information from the algorithms just described is propagated. If we allow these amounts to vary instead of fixing them through parameters $\varepsilon_{i j}$ and $\sigma_{i j}$, new sets of constraints can be formulated. We define variable $E_{i j}$ as the time at which task $i$ can start in unit $j \in \mathbf{J}_{i}$ and $S T_{i j}$ as the shortest time to the end of the horizon for task $i$ in unit $j \in \mathbf{J}_{i}$. We can readily write equation 28 to lower-bound these variables.

$E_{i j} \geq \varepsilon_{i j} ; S T_{i j} \geq \sigma_{i j} \forall i, j \in \mathbf{J}_{i}$

We can also generalize equation 20 to introduce these two new variables through equation 29.

$\sum_{t} \bar{\tau}_{i j} X_{i j t} \leq \eta-E_{i j}-S T_{i j} \quad \forall i, j \in J_{i}$

Consider the example shown in Figure 19. If we apply the TW algorithm to this network, we obtain $\varepsilon_{T 2, U 2}=\varepsilon_{T 3, U 2}=4$, since two batches of task T1 are required to start either T2 or T3. But, since T2 and T3 are performed in a common unit, only one of them can have an earliest start time of $4 h$, whereas the other will have an earliest start time of 6 or $7 \mathrm{~h}$. However, we do not know a priori which task will start first, especially if this network is a subsystem of a bigger network. We can then define variables $E_{T 2, U 2}$ and $E_{T 3, U 2}$, for which we know that feasible values are $\left(E_{T 2, U 2}, E_{T 3, U 2}\right)=$ $(4,6)$ or $\left(E_{T 2, U 2}, E_{T 3, U 2}\right)=(7,4)$. We conclude that a valid inequality for these variables is given by $E_{T 2, U 2}+E_{T 3, U 2} \geq 10$, which is stronger than the $\operatorname{set}\left\{E_{T 2, U 2} \geq \varepsilon_{T 2, U 2}=4, E_{T 3, U 2} \geq \varepsilon_{T 3, U 2}=4\right\}$

Moreover, we can define three different families of constraints based on the relative position of tasks within the network. Our goal is to generalize these concepts to more complex structures to obtain valid inequalities that can be used independently or coupled with the constraints derived in the previous section.

\subsection{Subsequent tasks}

Let us consider the case in which material $k$ is produced by task $i^{\prime}$ and consumed by task $i$. Then the earliest start time of task $i$ can be lower-bounded according to equation 30. We consider that this 
value has to be greater or equal than the sum of earliest start time for task $i^{\prime}$ and the time task $i^{\prime}$ takes to produce enough material to process the first batch of $i$.

$E_{i j} \geq E_{i^{\prime} j^{\prime}}+\bar{\tau}_{i^{\prime} j^{\prime}}\left\lceil\frac{\rho_{i k} \beta_{j}^{\text {min }}-\xi_{k 0}}{\rho_{i{ }^{\prime} k} \beta_{j^{\prime}}^{\max }}\right\rceil \begin{aligned} & \forall i, i^{\prime} \\ & \forall j \in \mathbf{J}_{i}, j^{\prime} \in \mathbf{J}_{i^{\prime}} \\ & \forall k \in \mathbf{K}_{i}^{-} \cap \mathbf{K}_{i^{\prime}}^{+}:\left|\mathbf{I}_{k}^{+}\right|=1, \xi_{k 0}<\rho_{i k} \beta_{j}^{\text {min }}\end{aligned}$

Similarly, equation 31 bounds the shortest tail of task $i^{\prime}$, using the processing time of task $i$.

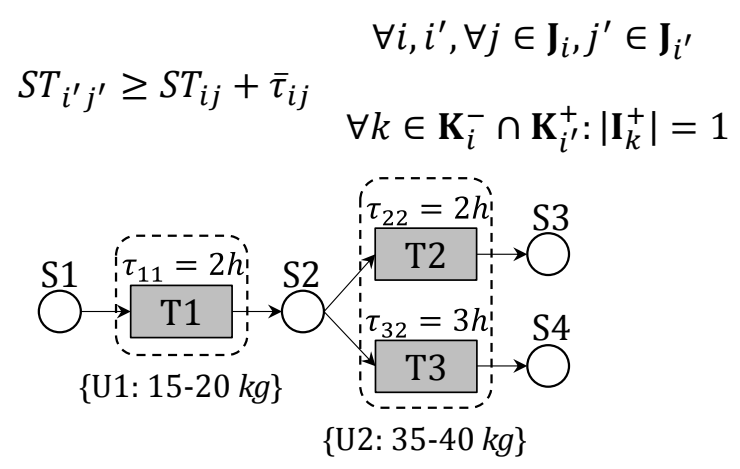

Figure 19. Small network to illustrate differences between constant and variable earliest start time.

\subsection{Tasks consuming the same material}

If two tasks $i$ and $i^{\prime}$ share a common input material $k$, its cumulative production limits the combinations of values of variable EST for both tasks. Let us consider the simple subsystem shown in Figure 20. If the capacity of unit U0 is such that one batch of task T0 is enough to start the first batch of both tasks T1 and T2 (e.g. U0: $60 \mathrm{~kg}$ ), then simple relations between the variable EST of T0, T1 and T2 are given by $E_{T 1} \geq E_{T 0}+\bar{\tau}_{T 0}$ and $E_{T 2} \geq E_{T 0}+\bar{\tau}_{T 0}$, which can be combined to write $E_{T 1}+E_{T 2} \geq 2\left(E_{T 0}+\bar{\tau}_{T 0}\right)$. On the other hand, when multiple batches of T0 are required to run the first batches of T1 and T2, then a careful analysis of the sequence and the required amounts has to be performed. For each sequence we can use equation 32 to find a valid inequality relating the EST variables. For the $(\mathrm{T} 1 \rightarrow \mathrm{T} 2)$ sequence we can write $E_{T 1} \geq E_{T 0}+\bar{\tau}_{T 0}\left[\rho_{T 1, S 1} \beta_{T 1}^{\min } / \rho_{T 0, S 1} \beta_{T 0}^{\max }\right\rceil=$ $E_{T 0}+2 \bar{\tau}_{T 0} \quad$ and $\quad E_{T 2} \geq E_{T 1}+\bar{\tau}_{T 0, U 0}\left[\rho_{T 2, S 1} \beta_{T 2}^{\min } / \rho_{T 0, S 1} \beta_{T 0}^{\max }\right]=E_{T 1}+4 \bar{\tau}_{T 0} \geq E_{T 0}+6 \bar{\tau}_{T 0}$. After combining we obtain $E_{T 1}+E_{T 2} \geq E_{T 0}+8 \bar{\tau}_{T 0}$. Similarly, the (T2 $\left.\rightarrow \mathrm{T} 1\right)$ sequence results in $E_{T 1}+E_{T 2} \geq E_{T 0}+10 \bar{\tau}_{T 0}$. In order to avoid cutting feasible integer solutions we choose the former (i.e. less restrictive) inequality as the valid constraint for the group $\{\mathrm{T} 1, \mathrm{~T} 2\}$.

Figure 21 shows the flowchart used to generalize this procedure to an arbitrary number $(n)$ of tasks sharing an input material. Note that we also choose to analyze subgroups of two and three tasks in order to add more valid inequalities to the constraint pool.

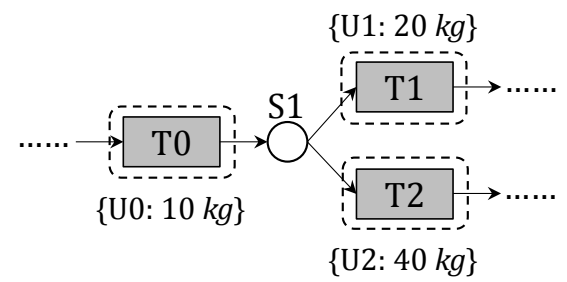

Figure 20. Simple subsystem with two tasks sharing an input material S1. 


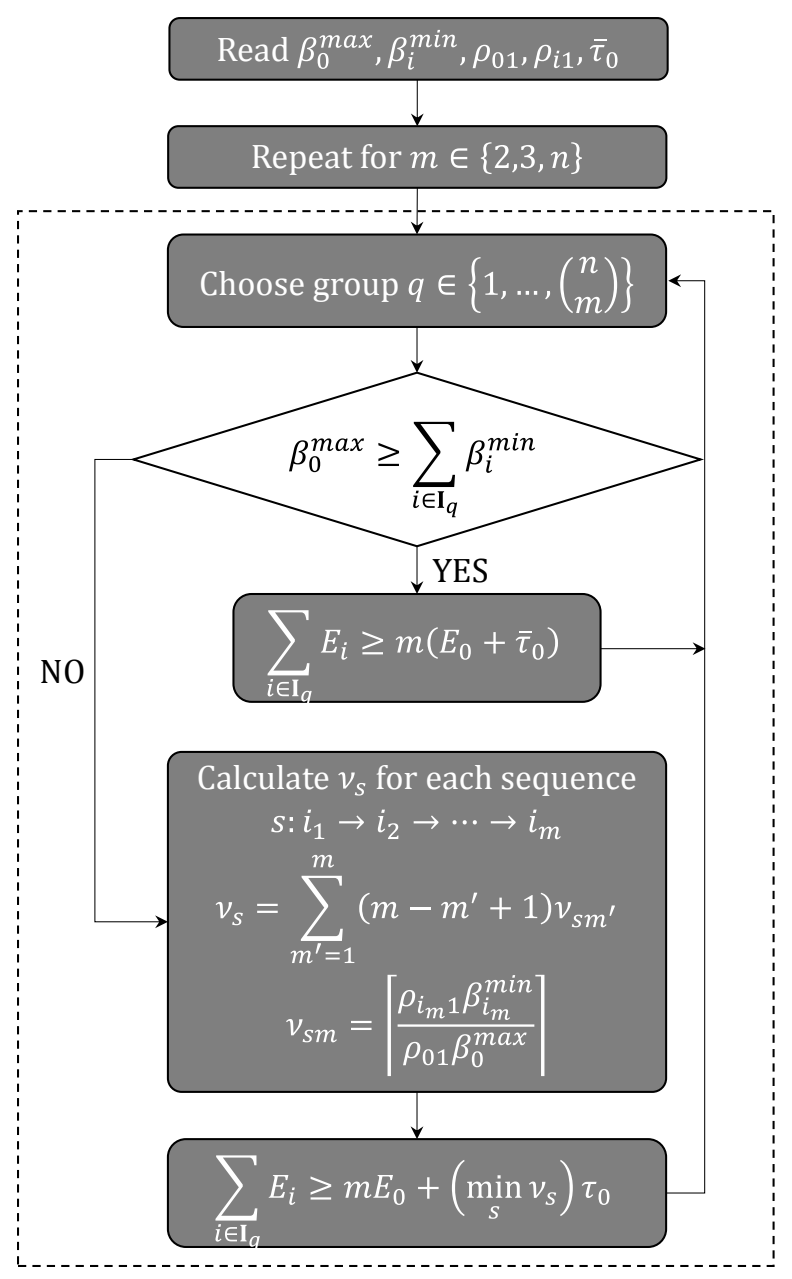

Figure 21. Valid inequalities for $\boldsymbol{n}$ tasks sharing an input material.

\subsection{Tasks sharing a common unit}

If $q \geq 2$ tasks share a common unit $j$, we consider the $q$ ! points obtained from considering the possible permutations of tasks and their respective processing times. For instance, when two tasks $i$ and $i^{\prime}$ are compatible with unit $j$, we need to consider the points $\left(\varepsilon_{i j}, \varepsilon_{i j}+\bar{\tau}_{i j}\right)$ and $\left(\varepsilon_{i^{\prime} j}+\right.$ $\left.\bar{\tau}_{i^{\prime} j}, \varepsilon_{i^{\prime} j}\right)$ for EST, and $\left(\sigma_{i j}, \sigma_{i j}+\bar{\tau}_{i j}\right)$ and $\left(\sigma_{i^{\prime} j}+\bar{\tau}_{i^{\prime} j}, \sigma_{i^{\prime} j}\right)$ for ST. We can then find the equation of the hyperplane on which these $q$ ! points lie in order to define the feasible half space whose inequality can be added to the pool of constraints. Equations 32 and 33 give the general form of such half spaces for variable EST and ST. Appendix C shows a sample calculation to illustrate this procedure.

$\sum_{i \in \mathbf{I}_{j}} v_{i j} E_{i j} \geq v_{j} \quad \forall j: \mathbf{I}_{j}$ is a type I group

$\sum_{i \in \mathbf{I}_{j}} \breve{v}_{i j} S T_{i j} \geq \breve{v}_{j} \quad \forall j: \mathbf{I}_{j}$ is a type I group

Where $v_{i j} / v_{j}\left(\breve{v}_{i j} / \breve{v}_{j}\right)$ are the coefficients of the EST (ST) hyperplane. 


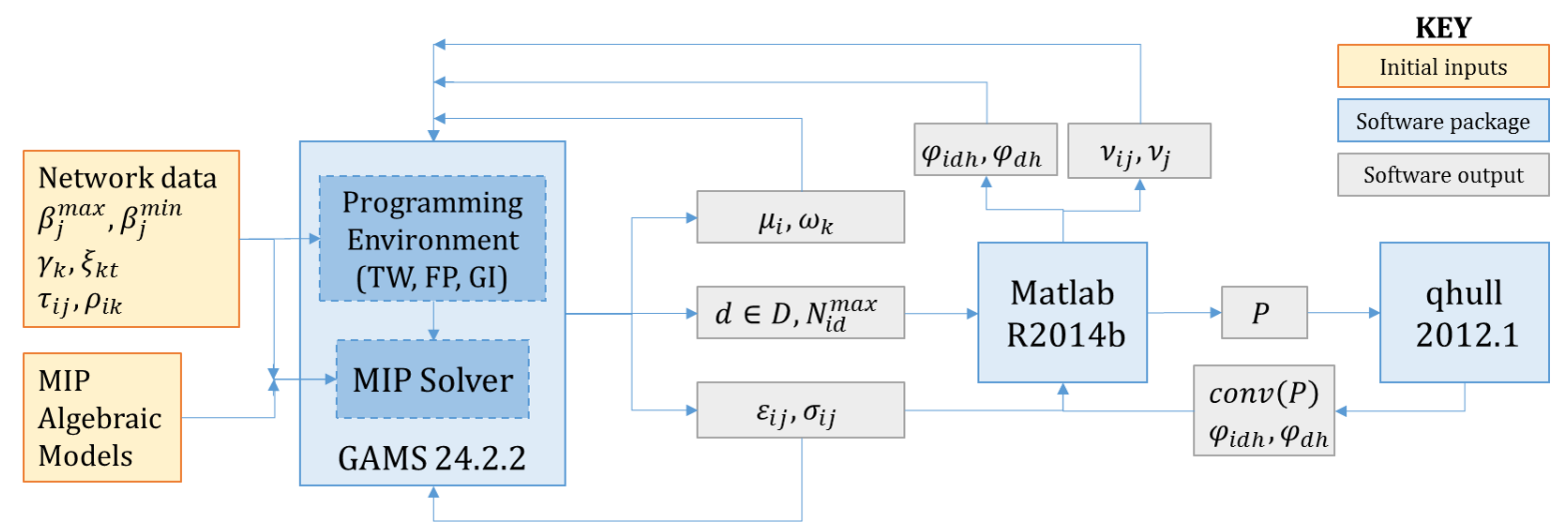

Figure 22. Flowchart for computational implementation.

Flow of information between the optimization engine (GAMS) and the numerical computing environment (Matlab) necessary to implement the proposed algorithms and the extension to variable EST.

\section{IMPLEMENTATION}

The computational implementation of the algorithms described in section 3 and the extensions presented in section 4 requires a dual interface using a numerical computing environment in addition to an optimization engine. The numerical computing environment we use is Matlab R2014b and we primarily employ it to (1) efficiently generate the sets of points required for the FR algorithm and general EST hyperplanes, and (2) act as the master environment to make calls to the qhull package (Barber et al., 1996) as part of the FR algorithm. The optimization engine we use is Cplex 12.6 on GAMS 24.2.

Figure 22 shows the interaction and the flow of information between the different computational engines required to implement the algorithms. The original network data and mathematical models are directly written in GAMS. In addition, the first three algorithms (TW, FP and GI) are also coded in GAMS to take advantage of its powerful set/index manipulation. Then two separate scripts in Matlab are used to execute the FR algorithm and the generation of hyperplanes for the variable EST case. First, the data on dependent sets of tasks is used as input to Matlab to generate the set of points $P$ satisfying equations $20-23$. This set is then used as the input to qhull to generate an explicit set of inequalities (equation 29) that defines $\operatorname{conv}(P)$, which is sent back to Matlab to write the final output file containing the coefficients of each inequality in GAMS format. Second, the data on earliest start time and shortest tail is passed to Matlab, which calculates the coefficients for the equations described in sections 4.2 and 4.3. Then it uses its built-in singular value decomposition (SVD) to calculate the equation of the hyperplane that contains all the points. The collinearity of the points is accepted without a formal proof, but it is verified by checking that the smallest singular value vanishes. The exchange of information between different packages is made using binary text files written by the software acting as master in each procedure.

In general, other than the solution of the MIP itself, the most expensive step is the generation and communication of the set of points $P$ that satisfy the criteria associated with a particular type of dependence in the FR algorithm. This is due to the combinatorial nature of the problem of generating all the possible permutations of number of batches for tasks within a group. Moreover, the computational effort increases as the number of tasks in the group and/or the scheduling horizon increase. Also, note that one of the most time-consuming tasks in this process is the writing of the binary files to exchange information among the different pieces of software, particularly 
between Matlab and GAMS. That is why any internal data manipulation within a given software should be done using native variables instead of writing extra binary files to avoid artificial extra time in the preprocessing stage. On average, when the FR algorithm is used, $70 \%$ of the preprocessing time is spent in GAMS and 30\% in Matlab/qhull. A possible way to improve computational efficiency is to solve the LP problems that appear in the preprocessing using Matlab.

\section{COMPUTATIONAL STUDY}

To determine the effectiveness of the proposed methods, we test them on 27 instances of three different networks with different scheduling horizons and using the models presented in section 2.2. Networks 1 and 2 (PN1 and PN2) are modified from Papageorgiou and Pantelides (Papageorgiou and Pantelides, 1996) and network 3 (PN3) is from Maravelias and Papalamprou (Maravelias and Papalamprou, 2009). Table 1 gives a summary of all the instances and contains the step size $\delta$ for the discrete-time model SP\&S, as well as the number of points, $N^{*}$, used to represent the best solution, which was determined through an iterative procedure. For this calculation, we used the solution obtained by the discrete-model as lower bound and iteratively increased the number of points in the continuous-time formulation until the optimal solution was found. The STN representations and corresponding data for all networks are available in the Supporting Information.

Table 1. Network, horizon and model for each computational run. A total of 27 instances are studied.

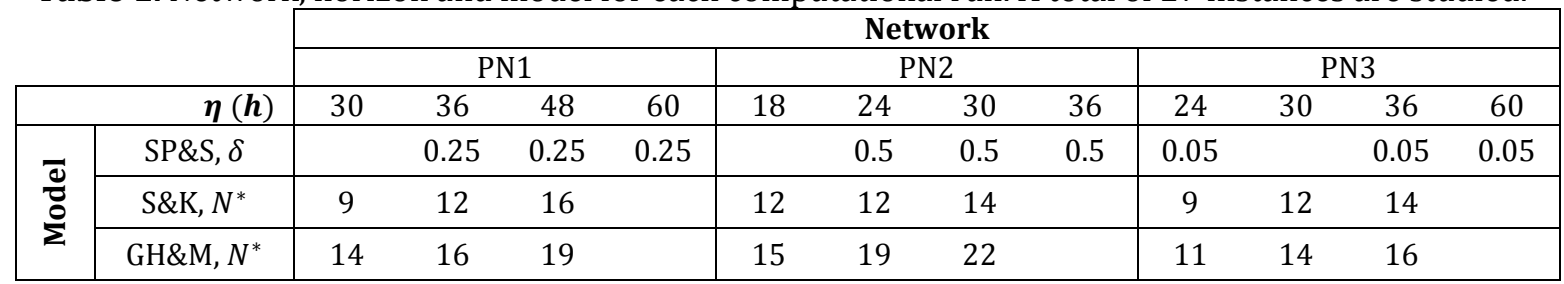

Note that the instances used for the discrete-time model are more difficult than those for the continuous-time models, since they are defined for longer horizon values.

The objective function we consider is profit maximization (equation 34). For the revenue term we use the selling price for each final product, $\pi_{k}$, while for the cost term we only consider a marginal production cost associated with the execution of task $i$ in unit $j \in \mathbf{J}_{i}, \alpha_{i j}=1 \times 10^{-4}$. This small value penalizes unnecessary task executions that do not lead to production of final products.

Profit $=\sum_{k \in \mathbf{K}} \sum_{t \in \mathbf{T}} \pi_{k} S_{k t}-\sum_{i \in \mathbf{I}} \sum_{j \in \mathbf{J}_{i}} \sum_{t \in \mathbf{T}} \alpha_{i j} X_{i j t}$

We analyze the effects of the various algorithms and the extension to variable EST and ST by defining four different formulations as shown in Table 2. Formulation F0 is the original model. Formulation F1 introduces the tightening from the four algorithms, using the results for the convex hull of the set of feasible number of task occurrences. Formulation F2 adds the tightening constraints from the variable earliest start time and shortest tail as discussed in section 4 . Formulation F3 is a combination of both strategies. Each formulation F0-F3 is applied to each of the 27 instances defined in Table 1, for a total of 108 runs.

We used GAMS 24.2.2/CPLEX 12.6 and Matlab R2014b on a computer with 8 GB of RAM and a 2.67 GHz Intel Core (i7-920) processor running on Windows 7. Default CPLEX settings, including cuts, 
are used. A time limit of one hour is enforced for all the runs. Complete model and solution statistics are given in the Supporting Information.

Table 2. Definition of the four proposed formulations.

\begin{tabular}{ll}
\hline Formulation & Equations \\
\hline F0 & SP\&S: 1-3 \\
& S\&K: A1-A14 \\
& GH\&M: A15-A29 \\
F1 & Discrete-time: F0+26-29 \\
& Continuous-time: F0+22,25,28,29 \\
F2 & F0+30-37 \\
F3 & F1+F2 \\
\hline
\end{tabular}

\subsection{Results}

The first measure we use to analyze the effectiveness of our methods is the number of runs solved to optimality. Approximately $52 \%$ of the total 108 runs found a solution and prove optimality within the time limit. In order to investigate the effect of the specific MIP model, Figure 23 presents the fraction of runs solved to optimality by each of the three models we consider.

Formulation F1 appears to solve more instances to optimality than any other formulation, regardless of the MIP model. Moreover, the discrete-time model proves to be more effective in finding optimal solutions with $90 \%$ of the runs solved to optimality when formulation F1 is used. Note that network PN2 represents a medium-scale process with 19 tasks and 27 materials and network PN3 uses a small step size, which significantly increases the number of binary variables and constraints in the SP\&S model. On the other hand, formulation F2 has no practical improvement with respect to the original models, in fact the lines for F0 and F2 completely overlap in Figure 23.

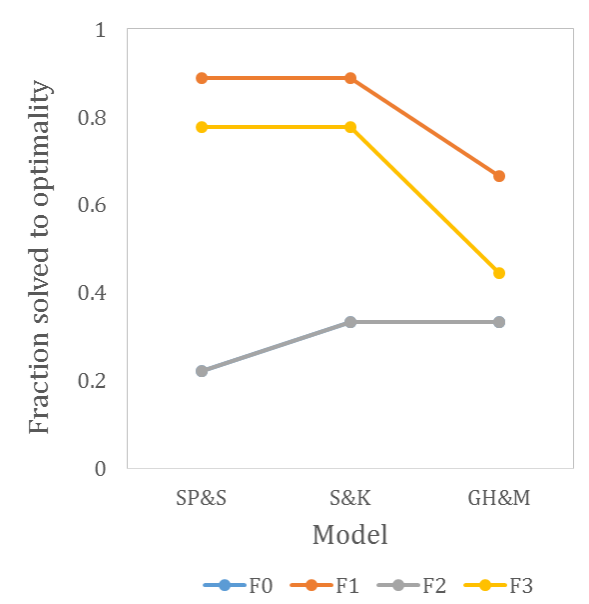

Figure 23. Fraction of runs solved to optimality using models SP\&S, S\&K, and GH\&M.

In industrial applications it is often sufficient to obtain a "good enough" feasible solution, rather than a provably optimal solution. In such cases, the effectiveness of a model can be assessed based on the optimality gap. Figure 24 contains results for all three models in terms of optimality gap. Instances are classified into three categories: (1) instances solved to optimality by all formulations, (2) instances solved to optimality by one or more of the new formulations, and (3) instances that reach the time limit with nonzero optimality gap with all formulations. Figure 24 shows that introducing any of the three proposed formulations brings advantages in terms of both 
computational time and optimality gap. Figure 24(a) shows that formulations F1 and F3 coupled with the discrete-time model produce the best results, in accordance with Figure 23. Figure 24(b) shows that F1 and F3 are the most effective for model S\&K; they solve to optimality five more instances than F0 and F2, and lead to the lowest optimality gap for the unsolved instance. Figure 24(c) shows the same pattern for formulations F1 and F3 with model GH\&M, but in addition, reveals a significant reduction in optimality gap, much more pronounced than model S\&K. Figures 24 (b) and (c) also show that model S\&K outperforms model GH\&M in all the instances. Model S\&K with formulations F1 and F3 solves more instances to optimality than its model GH\&M counterpart, reducing their average solution times. Instances not solved to optimality also exhibit a significant reduction in optimality gap with model S\&K. Clearly, all proposed formulations have a significant impact on reducing optimality gap for instances that reach the time limit. From the analysis presented in Figures 23 and 24, we establish that formulation F1 applied to model SP\&S leads to the best results in terms of consistency and decreasing both computational time and gap.

Another measure to show the effectiveness of a given method is the integrality gap defined as the relative difference between the optimal objective function values of the LP relaxation and the MIP model. Table 3 presents the results for each model and different formulations. Interestingly, the addition of the tightening constraints des not reduce the optimality gap of model SP\&S, although as discussed earlier, the reduction in computational time and optimality gap is significant. For the continuous-time models S\&K and GH\&M we observe once again that formulations F1 and F3 provide the greatest tightening when compared to their respective original formulations.
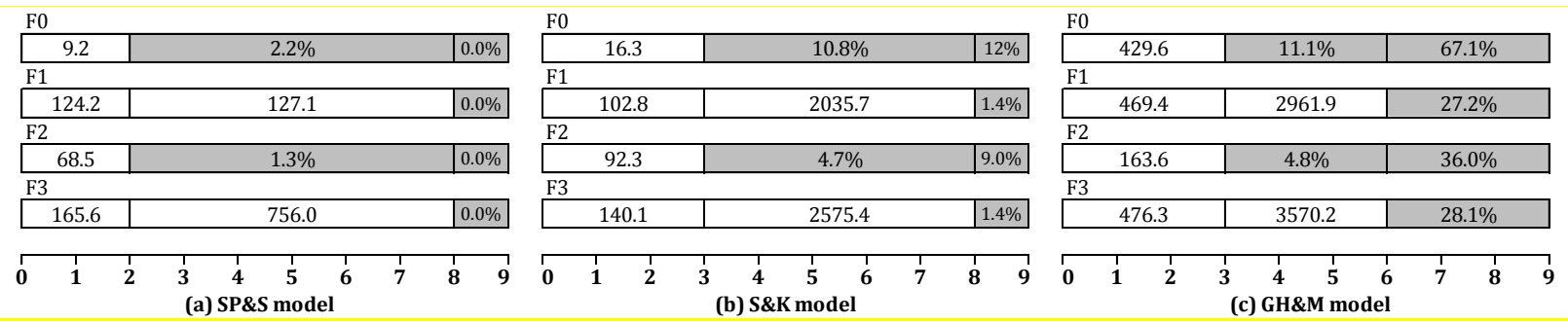

Figure 24. Average solution time or optimality gap.

Instances are solved using (a) discrete-time model SP\&S, and continuous-time models (b) S\&K, and (c) GH\&M.

Table 3. Average integrality gap (\%) for models SP\&S, S\&K, GH\&M and formulations F0-F3.

\begin{tabular}{cccc}
\hline Formulation & SP\&S & S\&K & GH\&M \\
\hline F0 & 9.0 & 37.5 & 51.6 \\
F1 & 9.0 & 18.6 & 22.6 \\
F2 & 9.0 & 27.8 & 39.9 \\
F3 & 9.0 & 18.6 & 22.6 \\
\hline
\end{tabular}




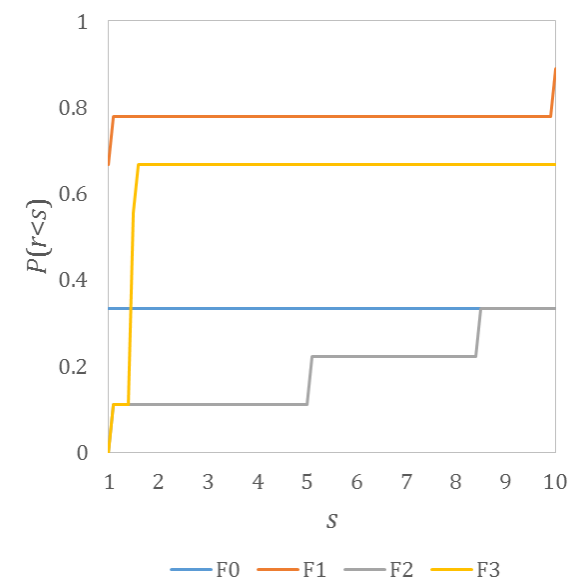

Figure 25. Performance chart for model SP\&S and proposed methods.

The abscissa represents the ratio (CPU time)/(CPU fastest instance) for each instance. The ordinate is the fraction of instances solved faster than the relative time in the abscissa.

In Figure 25, we present a performance chart for model SP\&S which has been found to be the most effective. The chart shows that formulation F1 is the most effective - 80\% of the instances are solved within 1.5 times the solution time of the fastest instance. The untightened model solves only $33 \%$ of the instances within one order of magnitude of the fastest instance, whereas formulations F2 and F3 solve 33\% and 67\% respectively within the same time.

Finally we calculate an improvement factor (IF) which is the ratio of the computational times of the original model and the best proposed formulation. The overall value (i.e., the average over all networks, horizons and models) is 15.2. The biggest improvement comes from the discrete-time model ( IF = 32.4), which is much higher than the factors of 2.7 and 7.0 for models S\&K and GH\&M respectively.

\section{INDUSTRIAL EXAMPLE}

In this section we present the application of the proposed methods to an industrial-scale instance. We use a simplified version of the problem introduced by (Nie et al., 2014) and shown in Figure 26. The process consists of five main product lines, A-E, that go through six common steps. Step 1 is a batch process for which two identical units are available (UB1, UB2). Steps 2, 3, and 5 are buffer tanks (UT1, UT2, UT3) and steps 4 and 6 are continuous processes (UC1, UC2). An intermediate F is required for lines $\mathrm{A}-\mathrm{C}$ and is produced by a single execution of the batch operation. In addition, the capacity of the second continuous operation is reduced with each execution; therefore a replenishing task must be run when the capacity falls below $25 \%$ of the maximum. Figure 27 is the STN representation of this process. We do not consider the changeovers included in the original reference. A scheduling horizon of five days (120 hours) is used with a scheduling period of 1 hour. We use the discrete-time model SP\&S and formulation F1 to solve this problem. Table 4 provides a summary of the results and Figure 28 contains the Gantt chart of the optimal schedule. 


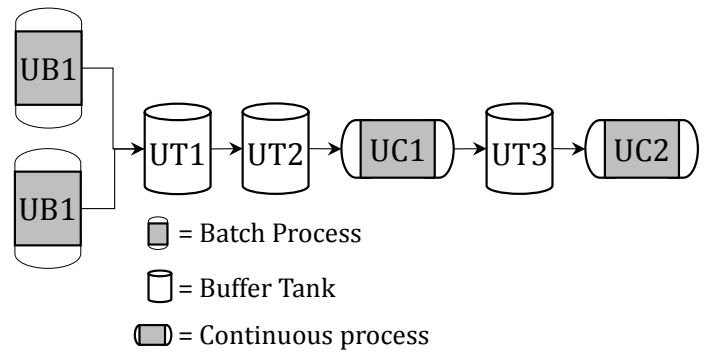

Figure 26. The Dow Process used as industrial-scale instance.

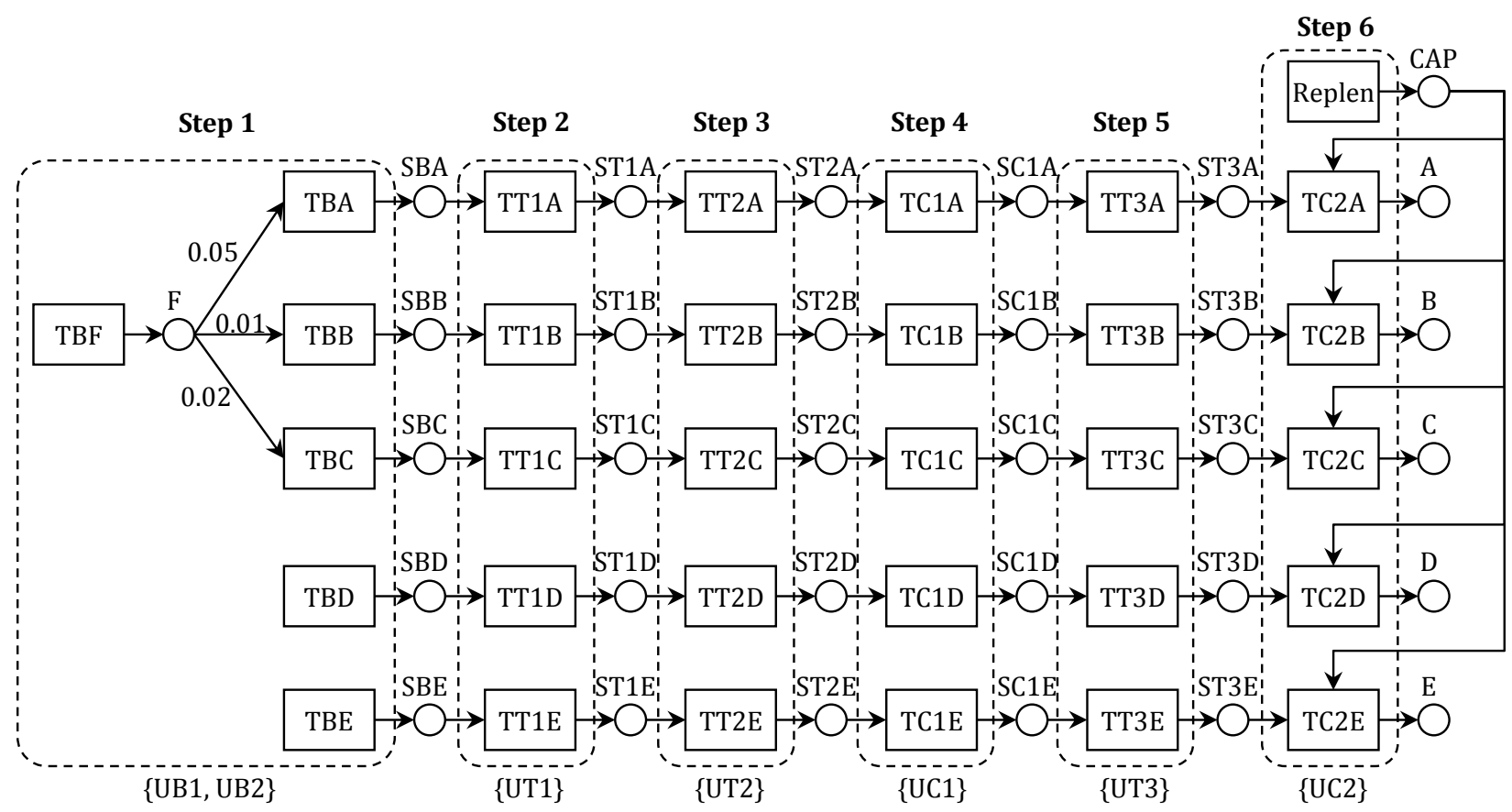

Figure 27. STN representation of the Dow Process.

Table 4. Comparison of results of original formulation and best proposed formulation on the Dow Example

\begin{tabular}{lcc} 
& F0 & F1 \\
\hline Constraints & 17,828 & 17,968 \\
Discrete variables & 4,598 & 4,682 \\
Total variables & 13,109 & 13,193 \\
Integer solution $(U S D)$ & $15,339.79$ & $15,339.79$ \\
LP relaxation solution $(U S D)$ & $15,406.46$ & $15,406.46$ \\
Preprocessing time $(s)$ & - & 678.91 \\
MIP solution time $(s)$ & 3,600 & 31.24 \\
Total CPU time $(s)$ & 3,600 & 710.15 \\
Gap (\%) & 0.43 & 0.00 \\
\hline
\end{tabular}

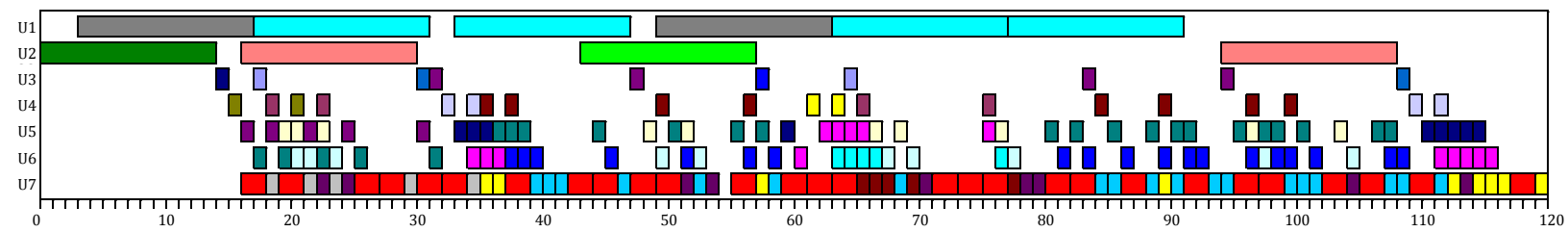

Figure 28. Gantt chart of optimal solution to the Dow example. 


\section{CONCLUSIONS}

We developed new methods for the enhancement of the solution of Mixed-Integer Programming models for chemical production scheduling. These methods consist of four preprocessing algorithms based on instance-specific information (network structure, recipe, processing times, initial inventory, and unit capacities) to calculate parameters that are then used to generate tightening constraints; as well as some new constraints based on variable earliest start times and shortest tails.

The Time Window (TW) algorithm calculates the effective time window that is available for each task to run, based on time and inventory restrictions. The Forward-Propagation (FP) algorithm calculates parameters to define the maximum feasible production for each task and material, and identifies whether a task is limited by its time window or the availability of its input materials. The Group Identification (GI) algorithm classifies tasks that share a common feature into groups, which in turn can be used to predict sets of feasible combinations of batches of these tasks. Finally, the Feasible Region (FR) algorithm uses the groups derived above to generate valid inequalities from the convex hull of the aforementioned set of points.

The proposed methods are applicable to all material-based, time-indexed, MIP scheduling models, discrete- and continuous-time. We show that the constraints derived from the sequence of preprocessing algorithms (formulation F1) produce the best results for both discrete- and continuous-time models. In many case, up to two orders of magnitude decrease in computational time is achieved when these constraints are applied to the discrete-time model. Finally, we were able to solve to optimality a large-scale instance and we expect that similar enhancements can be achieved in other industrial-scale instances.

\section{ACKNOWLEDGEMENTS}

The authors acknowledge financial support from the National Science Foundation under Grant CBET-1066206 and the American Chemical Society - Petroleum Research Fund under Grant 53313ND9. 


\section{NOTATION}

Sets/indices

$d \in \mathbf{D}$ Groups of dependent tasks

$i \in \mathbf{I} \quad$ Tasks

$j \in \mathbf{J} \quad$ Units

$h \in \mathbf{H}_{d}$ Equations that define the convex hull of points that characterize group $d$

$k \in \mathbf{K}$ Materials

$n \in \mathbf{N}$ Time points in continuous-time models

$q \quad$ Number of tasks that share a common resource in variable EST/ST calculations

$t \in \mathbf{T}$ Time points in discrete-time models

$\bar{t} \in \overline{\mathbf{T}} \quad$ Time points in TW_EST step

$\mathbf{I}_{d} \quad$ Tasks that belong to group $d$

$\mathbf{I}^{\text {Exp }} \quad$ Tasks that remain to be explored in FP and GI algorithms

$\mathbf{I}_{j} \quad$ Tasks that can be executed in unit $j$

$\mathbf{I}_{q} \quad q$-tuples of tasks that share a common resource in variable EST/ST calculations

$\mathbf{I}_{k}^{+} / \mathbf{I}_{k}^{-} \quad$ Tasks that produce/consume material $k$

$\mathbf{J}_{d} \quad$ Units that belong to group $d$

$\mathbf{J}_{i} \quad$ Units that can process task $i$

$\mathbf{J}_{k}^{+} / \mathbf{J}_{k}^{-} \quad$ Units that produce/consume material $k$

$\mathbf{K}^{E x p} \quad$ Materials that remain to be explored in TW and FP algorithms

$\mathbf{K}^{F} \quad$ Set of raw materials

$\mathbf{K}_{i}^{+} / \mathbf{K}_{i}^{-}$Materials produced/consumed by task $i$

$\mathbf{K}_{j}^{+} / \mathbf{K}_{j}^{-}$Materials produced/consumed by unit $j$

$\mathbf{K}^{P} \quad$ Set of final products

$\mathbf{T}^{\text {Exp }}$ Time points that remain to be explored in TW algorithm

Parameters

$\alpha_{i j} \quad$ Production cost for task $i \in \mathbf{I}$ in unit $j \in \mathbf{J}_{i}$

$\beta_{j}^{\text {max }} / \beta_{j}^{\text {min }} \quad$ Maximum/minimum batch size for unit $j \in \mathbf{J}$

$\gamma_{k} \quad$ Storage capacity for material $k \in \mathbf{K}$

$\delta \quad$ Length (step size) of intervals in discrete-time models

$\varepsilon_{i j} \quad$ Earliest start time for task $i \in \mathbf{I}$ in unit $j \in \mathbf{J}_{i}$

$\varepsilon_{j} \quad$ Earliest start time for task $i \in \mathbf{I}$ in unit $j \in \mathbf{J}_{i}$

$\eta \quad$ Scheduling horizon

$\zeta_{i d}^{\max } \quad$ Maximum number of batches of task $i \in \mathbf{I}$ in group $d$

$\theta_{i j} \quad$ Time window for task $i \in \mathbf{I}$ in unit $j \in \mathbf{J}_{i}$

$\theta_{j} \quad$ Time window for unit $j \in \mathbf{J}$

$\lambda_{i j}^{S}$

Latest finish time for task $i \in \mathbf{I}$ in unit $j \in \mathbf{J}_{i}$ so that the intermediates it produces have enough time to be transformed into final products.

$\mu_{i} \quad$ Maximum cumulative production of task $i \in \mathbf{I}$ within the given horizon

$\mu_{i}^{1} \quad$ Corrected value of $\mu_{i}$ due to production intervals

$\mu_{i}^{L P} \quad$ Value of $\mu_{i}$ based on inventory restrictions modeled as an LP

$\mu_{i}^{T W} \quad$ Value of $\mu_{i}$ based on available time window

$v_{i j} / \breve{v}_{i j} \quad$ Coefficient for EST/ST constraints of task $i \in \mathbf{I}_{q}$ in unit $j \in \mathbf{J}_{i}$ 


\begin{tabular}{|c|c|}
\hline$v_{j} / \breve{v}_{j}$ & Right-hand-side coefficient for EST/ST constraints of unit $j \in \mathbf{J}$ \\
\hline$\xi_{k t}$ & Delivery of material $k \in \mathbf{K}$ at time $t$ \\
\hline$\pi_{k}$ & Unit price of material $k \in \mathbf{K}$ \\
\hline$\rho_{i k}$ & $\begin{array}{l}\text { Conversion coefficient for material } k \in \mathbf{K} \text { produced }\left(\rho_{i k}>0\right) \text { or consumed }\left(\rho_{i k}<0\right) \\
\text { by task } i \in \mathbf{I}\end{array}$ \\
\hline$\sigma_{i j}$ & Shortest tail for task $i \in \mathbf{I}$ in unit $j \in \mathbf{J}_{i}$ \\
\hline$\sigma_{j}$ & Shortest tail for unit $j \in \mathbf{J}$ \\
\hline $\bar{\tau}_{i j}$ & Processing time for task $i \in \mathbf{I}$ in unit $j \in \mathbf{J}_{i}$ \\
\hline$\tau_{i j}$ & $\begin{array}{l}\text { Processing time for task } i \in \mathbf{I} \text { in unit } j \in \mathbf{J}_{i} \text { as a multiple of the step size } \delta \text { for } \\
\text { discrete-time representation }\end{array}$ \\
\hline$v_{i j}$ & Batches of task $i \in \mathbf{I}_{k}^{-}$in unit $j \in \mathbf{J}_{i}$ required to calculate $\omega_{k}^{R}$ \\
\hline$\varphi_{i d h}$ & Linear combination coefficient for task $i \in \mathbf{I}_{d}$ of group $d$ in equation $h \in \mathbf{H}_{d}$ \\
\hline$\varphi_{d h}$ & Right-hand-side coefficient for group $d$ in equation $h \in \mathbf{H}_{d}$ \\
\hline$\phi_{i t}^{E}$ & Cumulative production for task $i \in \mathbf{I}$ in any unit starting at or before time $t$ \\
\hline$\phi_{k}^{S}$ & Minimum time required to process material $k \in \mathbf{K}$ so it leads to final products \\
\hline$\chi_{i j t}^{E}$ & Cumulative production for task $i \in \mathbf{I}$ in unit $j \in \mathbf{J}_{i}$ starting at or before time $t$ \\
\hline$\psi_{k t}^{E}$ & Maximum amount of material $k \in \mathbf{K}$ available at time $t$ \\
\hline$\omega_{k}$ & Maximum cumulative production of material $k \in \mathbf{K}$ within the given horizon \\
\hline$\omega_{k \breve{n}}^{R}$ & Required amount of input materials for $\breve{n}_{i j}$ batches of task $i \in \mathbf{I}_{k}^{-}$in unit $j \in \mathbf{J}_{i}$ \\
\hline \multicolumn{2}{|c|}{ Binary variables } \\
\hline$X_{i j t} / X_{i j n}$ & It is equal to one if task $i \in \mathbf{I}$ starts in unit $j \in \mathbf{J}_{i}$ at time $t \in \mathbf{T} / n \in \mathbf{N}$ \\
\hline$Y_{i j n}$ & It is equal to one if task $i \in \mathbf{I}$ finishes in unit $j \in \mathbf{J}_{i}$ at time $n \in \mathbf{N}$ \\
\hline \multicolumn{2}{|c|}{ Continuous nonnegative variables } \\
\hline$\hat{B}_{i j}$ & Cumulative production of task $i \in \mathbf{I}$ in unit $j \in \mathbf{J}_{i}$ \\
\hline$B F_{i j n} / B P_{i j n}$ & $\begin{array}{l}\text { Batch size of task } i \in \mathbf{I} \text { that starts/continues to be processed in unit } j \in \mathbf{J}_{i} \text { at time } \\
n \in \mathbf{N}\end{array}$ \\
\hline$B S_{i j t} / B S_{i j n}$ & Batch size of task $i \in \mathbf{I}$ that starts in unit $j \in \mathbf{J}_{i}$ at time $t \in \mathbf{T} / n \in \mathbf{N}$ \\
\hline$E_{i j}$ & Variable earliest start time for task $i \in \mathbf{I}$ in unit $j \in \mathbf{J}_{i}$ \\
\hline$N_{i}$ & Number of batches of task $i \in \mathbf{I}$ \\
\hline$Q_{i}$ & Amount produced by task $i \in \mathbf{I}$ \\
\hline$S T_{i j}$ & Variable shortest tail for task $i \in \mathbf{I}$ in unit $j \in \mathbf{J}_{i}$ \\
\hline $\begin{array}{l}S_{k t} / S_{k n} \\
T_{n}\end{array}$ & $\begin{array}{l}\text { Inventory level of material } k \in \mathbf{K} \text { at time point } t \in \mathbf{T} / n \in \mathbf{N} \text { with } S_{k 0}=\xi_{k} \\
\text { Actual value of time point } n \in \mathbf{N}\end{array}$ \\
\hline$W_{k}$ & Cumulative production of material $k \in \mathbf{K}$ \\
\hline
\end{tabular}




\section{APPENDIX A: Continuous-time Models}

\section{A.1. Model S\&K}

Model S\&K uses the following additional variables:

$Z_{j n}: \quad$ Binary. It equals one if any task begins in unit $j \in \mathbf{J}$ at time $n \in \mathbf{N}$

$Y P_{i j n}: \quad$ Binary. It equals one if task $i \in \mathbf{I}$ continues to be processed in unit $j \in \mathbf{J}_{i}$ at time $n \in \mathbf{N}$

$S L_{n}: \quad$ Duration of interval $n \in \mathbf{N} \backslash\{0\}$

$T R_{j n}$ : $\quad$ Continuous nonnegative. Time remaining in unit $j \in \mathrm{J}$ to complete an ongoing task at time $n \in \mathbf{N}$

Equations A1-A14 define the S\&K model.

$S L_{n}=T_{n}-T_{n-1} \quad \forall n \in \mathbf{N} \backslash\{0\}$

$\sum_{n \in \mathbf{N} \backslash\{0\}} S L_{n} \leq \eta$

$Z_{j n}=\sum_{i \in \mathbf{I}_{j}} X_{i j n} \quad \forall j \in \mathbf{J}, \forall n \in \mathbf{N} \backslash\{N\}$

$Z_{j n}=\sum_{i \in \mathbf{I}_{j}} Y_{i j n} \quad \forall j \in \mathbf{J}, \forall n \in \mathbf{N} \backslash\{0\}$

$Y P_{i j n}=Y P_{i j(n-1)}+X_{i j(n-1)}-Y_{i j n} \quad \forall i \in \mathbf{I}, \forall j \in \mathbf{J}_{i}, \forall n \in \mathbf{N} \backslash\{0, N\}$

$T R_{j(n+1)} \geq T R_{j n}+\sum_{i \in \mathbf{I}_{j}} \tau_{i j} X_{i j n}-S L_{(n+1)} \quad \forall j \in \mathbf{J}, \forall n \in \mathbf{N} \backslash\{N\}$

$B P_{i j n}=B P_{i j(n-1)}+B S_{i j(n-1)}-B F_{i j n} \quad \forall i \in \mathbf{I} \backslash\{0\}, \forall j \in \mathbf{J}_{i}, \forall n \in \mathbf{N} \backslash\{0\}$

$\beta_{j}^{\text {min }} X_{i j n} \leq B S_{i j n} \leq \beta_{j}^{\text {max }} X_{i j n} \quad \forall i \in \mathbf{I} \backslash\{0\}, \forall j \in \mathbf{J}_{i}, \forall n \in \mathbf{N}$

$\beta_{j}^{\text {min }} Y P_{i j n} \leq B P_{i j n} \leq \beta_{j}^{\max } Y P_{i j n} \quad \forall i \in \mathbf{I} \backslash\{0\}, \forall j \in \mathbf{J}_{i}, \forall n \in \mathbf{N}$

$\beta_{j}^{\text {min }} Y_{i j n} \leq B F_{i j n} \leq \beta_{j}^{\text {max }} Y_{i j n} \quad \forall i \in \mathbf{I} \backslash\{0\}, \forall j \in \mathbf{J}_{i}, \forall n \in \mathbf{N}$

$T R_{j n} \leq \sum_{i \in \mathbf{I}_{j}} \tau_{i j} Y P_{i j n} \quad \forall j \in \mathbf{J}, \forall n \in \mathbf{N} \backslash\{0, N\}$

$S_{k n}=S_{k(n-1)}+\sum_{i \in \mathbf{I}_{k}^{+}} \sum_{j \in \mathbf{J}_{i}} \rho_{i k} B F_{i j n}+\sum_{i \in \mathbf{I}_{k}^{-}} \sum_{j \in \mathbf{J}_{i}} \rho_{i k} B S_{i j n} \leq \gamma_{k} \quad \forall k \in \mathbf{K}, \forall n \in \mathbf{N}$

Finally, equations A13-A14 are required to impose bounds on variables.

$S L_{n} \leq \max _{j \in \mathbf{J}}\left[\max _{i \in \mathbf{I}_{j}}\left(\tau_{i j}\right)\right] \quad \forall n \in \mathbf{N} \backslash\{0\}$

$T R_{j n} \leq \max _{i \in \mathbf{I}_{j}}\left(\tau_{i j}\right) \quad \forall j \in \mathbf{J}, \forall n \in \mathbf{N}$

\section{A.2. Model GH\&M}


In order to explicitly write all the equations in model GH\&M, we define the following additional decision variables required for its implementation:

$E_{j n} / V_{j n}: \quad$ Binary. It equals one if unit $j \in \mathbf{J}$ is at execution/idle state during interval $n \in \mathbf{N} \backslash\{N\}$

$Z_{j n}: \quad$ Binary. It equals one if at time $n \in \mathbf{N}$, unit $j \in \mathbf{J}$ continues executing a task that started at a previous time point

$T_{j n}^{L B} / T_{j n}^{E E}: \quad$ Continuous nonnegative. Amount of time the beginning/end of a task is delayed/anticipated in unit $j \in \mathbf{J}$ with respect to its nominal beginning/end at time $n \in \mathbf{N}$

$T_{j n}^{V}$ : $\quad$ Continuous nonnegative. Time spent by unit $j \in \mathbf{J}$ in the idle state during interval $n \in \mathbf{N} \backslash\{N\}$

The model is defined through equations A15-A29.

$E_{j n}+V_{j n}=1 \quad \forall j \in \mathbf{J}, \forall n \in \mathbf{N} \backslash\{0\}$

$E_{j n}=Z_{j n}+\sum_{i \in \mathbf{I}_{j}} X_{i j n} \quad \forall j \in \mathbf{J}, \forall n \in \mathbf{N} \backslash\{0\}$

$Z_{j n}=Z_{j(n-1)}+\sum_{i \in \mathbf{I}_{j}} X_{i j(n-1)} \quad \forall j \in \mathbf{J}, \forall n \in \mathbf{N} \backslash\{0\}$

$T_{j n}^{L B} \leq \eta \sum_{i \in \mathbf{I}_{j} \backslash \mathbf{I}^{Z W-}} X_{i j n} \quad \forall j \in \mathbf{J}, \forall n \in \mathbf{N} \backslash\{0\}$

$T_{j n}^{E E} \leq \eta \sum_{i \in \mathbf{I}_{j} \backslash \mathbf{I}^{Z W+}} Y_{i j n} \quad \forall j \in \mathbf{J}, \forall n \in \mathbf{N} \backslash\{1\}$

$T_{j n}^{V} \leq \eta V_{j n} \quad \forall j \in \mathbf{J}, \forall n \in \mathbf{N} \backslash\{N\}$

$T_{n+1}-T_{n}-\eta\left(1-V_{j n}\right) \leq T_{j n}^{V} \leq T_{n+1}-T_{n} \quad \forall j \in \mathbf{J}, \forall n \in \mathbf{N} \backslash\{N\}$

$T_{n} \geq \sum_{1<n^{\prime} \leq n}\left[T_{j n^{\prime}}^{E E}+\sum_{i \in \mathbf{I}_{j}}\left(\tau_{i j}^{F} Y_{i j n^{\prime}}+\tau_{i j}^{V} B F_{i j n^{\prime}}\right)\right]+\sum_{n^{\prime}<n}\left(T_{j n^{\prime}}^{L B}+T_{j n^{\prime}}^{V}\right) \quad \forall j \in \mathbf{J}, \forall n \in \mathbf{N} \backslash\{N\}$

$\eta-T_{n} \geq \sum_{n \leq n^{\prime}<N}\left[T_{j n^{\prime}}^{L B}+T_{j n^{\prime}}^{V}+\sum_{i \in \mathbf{I}_{j}}\left(\tau_{i j}^{F} X_{i j n^{\prime}}+\tau_{i j}^{V} B S_{i j n^{\prime}}\right)\right]+\sum_{n^{\prime}>n} T_{j n^{\prime}}^{E E} \quad \forall j \in \mathbf{J}, \forall n \in \mathbf{N} \backslash\{N\}$

$\eta=\sum_{n>1}\left[T_{j n}^{E E}+\sum_{i \in \mathbf{I}_{j}}\left(\tau_{i j}^{F} Y_{i j n}+\tau_{i j}^{V} B F_{i j n}\right)\right]+\sum_{n<N}\left(T_{j n}^{L B}+T_{j n}^{V}\right) \quad \forall j \in \mathbf{J}$

$\beta_{j}^{\text {min }} X_{i j n} \leq B S_{i j n} \leq \beta_{j}^{\max } X_{i j n} \quad \forall i \in \mathbf{I}, \forall j \in \mathbf{J}_{i}, \forall n \in \mathbf{N}$

$\beta_{j}^{\text {min }} Y_{i j n} \leq B F_{i j n} \leq \beta_{j}^{\text {max }} Y_{i j n} \quad \forall i \in \mathbf{I}, \forall j \in \mathbf{J}_{i}, \forall n \in \mathbf{N}$

$\beta_{j}^{\text {min }} Z_{j n} \leq \sum_{i \in \mathbf{I}_{j}} B P_{i j n} \leq \beta_{j}^{\max } Z_{j n} \quad \forall j \in \mathbf{J}, \forall n \in \mathbf{N}$ 


$$
\begin{aligned}
& B S_{i j n}+B P_{i j n}=B P_{i j(n+1)}+B F_{i j(n+1)} \quad \forall i \in \mathbf{I}, \forall j \in \mathbf{J}_{i}, \forall n \in \mathbf{N} \backslash\{N\} \\
& S_{k n}=S_{k(n-1)}+\sum_{i \in \mathbf{I}_{k}^{+}} \sum_{j \in \mathbf{J}_{i}} \rho_{i k} B F_{i j n}+\sum_{i \in \mathbf{I}_{k}^{-}} \sum_{j \in \mathbf{J}_{i}} \rho_{i k} B S_{i j n} \leq \gamma_{k} \quad \forall k \in \mathbf{K}, \forall n \in \mathbf{N}
\end{aligned}
$$

\section{APPENDIX B: Calculation of $\boldsymbol{\mu}_{\boldsymbol{i}}^{\mathbf{1}}$}

For a given task $i$, let us define the set $m \in \mathbf{M}_{i}$ to index the production intervals that result when different combinations of units processing task $i$ are considered. Let us also define $\epsilon_{j}^{m}$ as the number of batches of task $i$ processed in unit $j \in \mathbf{J}_{i}$ for interval $m$. The maximum value $\epsilon_{j}^{m}$ takes is obtained by rounding up the ratio of the cumulative production of task $i$ to the maximum capacity of unit $j \in \mathbf{J}_{i}$, i.e. $\epsilon_{j}^{\max }=\left\lceil\mu_{i} / \beta_{j}^{\max }\right\rceil$. Then, equation B1 provides the number of elements in the set $\mathbf{M}_{i}$, where the first term represents combinations with $\epsilon_{j}^{m} \in\left[0, \epsilon_{j}^{\max }-1\right]$ and the second term is the number of intervals with $\epsilon_{j}^{m}=\epsilon_{j}^{\max }$ and $\epsilon_{j^{\prime}}^{m}=0 \forall j^{\prime} \neq j$, i.e. the combinations that contain only one unit.

$$
\left|\mathbf{M}_{i}\right|=\prod_{j \in \mathbf{J}_{i}} \epsilon_{j}^{\max }+\left|\mathbf{J}_{i}\right|
$$

Equation B2 defines the corrected value for the feasible cumulative production of task $i$.

$$
\mu_{i}^{1}=\left\{\begin{array}{cc}
\mu_{i} & \text { if } \exists m: \sum_{j \in \mathbf{J}_{i}} \epsilon_{j}^{m} \beta_{j}^{m i n} \leq \mu_{i} \leq \sum_{j \in \mathbf{J}_{i}} \epsilon_{j}^{m} \beta_{j}^{\text {max }} \\
\max _{m \in \mathbf{M}_{i}}\left\{\sum_{j \in \mathbf{J}_{i}} \epsilon_{j}^{m} \beta_{j}^{\max }: \sum_{j \in \mathbf{J}_{i}} \epsilon_{j}^{m} \beta_{j}^{m a x} \leq \mu_{i}\right\} & \text { otherwise }
\end{array}\right.
$$

As an illustration let us consider a task T1 that has a maximum cumulative production of $70 \mathrm{~kg}$ and is compatible with units $\mathrm{U} 1$ and $\mathrm{U} 2$, with capacities $\beta_{U 1}^{\min }=25 \mathrm{~kg}, \beta_{U 1}^{\max }=30 \mathrm{~kg}, \beta_{U 2}^{\min }=$ $55 \mathrm{~kg}, \beta_{U 2}^{\max }=60 \mathrm{~kg}$. First, we calculate $\epsilon_{U 1}^{\max }=\lceil 70 / 30\rceil=3$ and $\epsilon_{U 2}^{\max }=\lceil 70 / 60\rceil=2$. Then, using equation B1 we obtain the total number of intervals $\left|\mathbf{M}_{T 1}\right|=8$. Figure B1 presents a complete analysis of the feasible intervals, based on combinations of U1 and U2 and the expressions included in equation 20. Since $\mu_{T 1}=70$ does not fall in any of the intervals, equation B2 uses the upper bound of the closest interval to obtain $\mu_{T 1}^{1}=60$. 


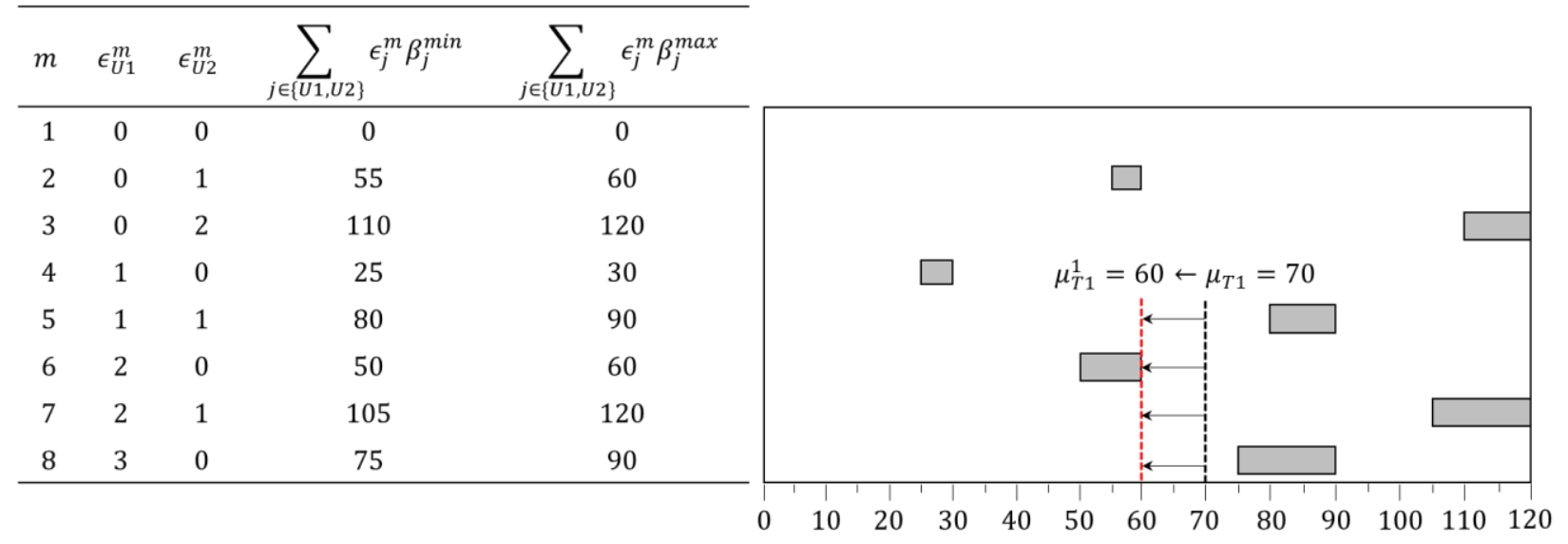

Figure B1. Production intervals for combinations of two units U1 and U2 with capacities [25,30] and [55,60]. A task T1 compatible with these units cannot have a maximum cumulative production of $\mu_{T 1}=70$. From the feasible production intervals we obtain a corrected value $\mu_{T 1}^{1}=60$.

\section{APPENDIX C: Hyperplane equation for variable EST}

Let us consider the network depicted in Figure C1 that consists of three tasks $\{\mathrm{T} 1, \mathrm{~T} 2, \mathrm{~T} 3\}$ with earliest start time of 2 hours sharing a common unit U1. The values for processing times are $\tau_{T 1, U 1}=10, \tau_{T 2, U 1}=4, \tau_{T 3, U 1}=2$. Since the three tasks share unit U1, only one of the EST values will be effectively enforced, and the other two tasks will need to wait until the unit is available. For instance, if T1 is executed first its variable value of EST can be set to $E_{T 1, U 1}=2=\varepsilon_{T 1, U 1}$. If task T2 follows, it has to wait for 10 hours until U1 is available. Therefore we can set $E_{T 2, U 1}=12=\varepsilon_{T 1, U 1}+$ $\bar{\tau}_{T 1, U 1}$. Finally, task T3 has to wait an additional 4 hours to be executed and its EST can be set to $E_{T 3, U 1}=16=\varepsilon_{T 1, U 1}+\bar{\tau}_{T 1, U 1}++\bar{\tau}_{T 2, U 1}$. Clearly, the values of $E_{i j}$ are highly dependent on the permutation in the order of tasks. Table C1 shows the feasible values of $E_{i j}$ that can be set depending on the task sequencing.

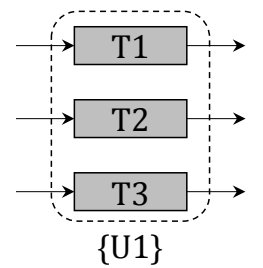

Figure C1. Illustrative network for EST hyperplane generation.

Table C1. Permutations of tasks sharing unit $\mathrm{U} 1$ and corresponding variable EST values.

\begin{tabular}{ccccccc}
\hline Sequence & T1-T2-T3 & T1-T3-T2 & T2-T3-T1 & T2-T1-T3 & T3-T1-T2 & T3-T2-T1 \\
\hline$E_{T 1, U 1}$ & 2 & 2 & 8 & 6 & 4 & 8 \\
$E_{T 2, U 1}$ & 12 & 14 & 2 & 2 & 14 & 4 \\
$E_{T 3, U 1}$ & 16 & 12 & 6 & 16 & 2 & 2 \\
\hline$\sum_{i} E_{i, U 1}$ & 30 & 28 & 16 & 24 & 20 & 14 \\
\hline
\end{tabular}

From Table C1 it is clear that $E_{T 1, U 1}+E_{T 2, U 1}+E_{T 3, U 1} \geq 14$ is a valid constraint. However, we can obtain the tightest possible inequality, by finding the hyperplane that best represents these six points defined by different permutations of tasks. Let us define matrix $A$ as the array of point coordinates resulting from the permutation of tasks. For this particular instance, 


$$
A=\left[\begin{array}{ccc}
2 & 12 & 16 \\
2 & 14 & 12 \\
8 & 2 & 6 \\
6 & 2 & 16 \\
4 & 14 & 2 \\
8 & 4 & 2
\end{array}\right]
$$

We then normalize the points to force the plane to contain the origin, by subtracting the vector of average coordinates from each column $b=\left[\begin{array}{lll}5 & 8 & 9\end{array}\right]$ to obtain:

$$
\bar{A}=\left[\begin{array}{ccc}
-3 & 4 & 7 \\
-3 & 6 & 3 \\
3 & -6 & -3 \\
1 & -6 & 7 \\
-1 & 6 & -7 \\
3 & -4 & -7
\end{array}\right]
$$

Finally the SVD of $\bar{A}^{\prime} \bar{A}=U \Sigma V^{\prime}$ provides the necessary information in terms of collinearity and equation of the interpolating hyperplane. If we find that $\min \left\{\sigma: \sigma\right.$ is a singular value of $\left.\bar{A}^{\prime} \bar{A}\right\}=\Sigma_{33}=$ 0 (such as in this example), then we conclude that all the points belong to a single hyperplane. The corresponding singular column vector $u_{3}=v_{3}$ provides the coefficients of the hyperplane equation. Application to the vector of mean coordinates $b$ results in the right hand side of the equation. Using Matlab we obtain the final inequality $5 E_{T 1, U 1}+2 E_{T 2, U 1}+E_{T 3, U 1} \geq 50$ that corresponds to the tightest constraint we can enforce on the permutation of tasks (i.e. equation 32 with $v_{T 1, U 1}=$ $5, v_{T 2, U 1}=2, v_{T 3, U 1}=1, v_{U 1}=50$ ). If $\Sigma_{33}>0$, then the points are not collinear and then we use the inequality derived from table $\mathrm{C} 1$. 


\section{REFERENCES}

Barber, C. B., D. P. Dobkin and H. Huhdanpaa (1996). The Quickhull algorithm for convex hulls. Acm Transactions on Mathematical Software 22(4): 469-483.

Bassett, M. H., J. F. Pekny and G. V. Reklaitis (1996). Decomposition techniques for the solution of large-scale scheduling problems. Aiche Journal 42(12): 3373-3387.

Baumann, P. and N. Trautmann (2013). A continuous-time MILP model for short-term scheduling of makeand-pack production processes. International Journal of Production Research 51(6): 1707-1727.

Burkard, R. E. and J. Hatzl (2005). Review, extensions and computational comparison of MILP formulations for scheduling of batch processes. Computers \& Chemical Engineering 29(8): 1752-1769.

Castro, P., A. P. F. D. Barbosa-Povoa and H. Matos (2001). An improved RTN continuous-time formulation for the short-term scheduling of multipurpose batch plants. Industrial \& Engineering Chemistry Research 40(9): 2059-2068.

Castro, P. M., M. Erdirik-Dogan and I. E. Grossmann (2008). Simultaneous batching and scheduling of single stage batch plants with parallel units. Aiche Journal 54(1): 183-193.

Castro, P. M. and I. E. Grossmann (2005). New continuous-time MILP model for the short-term scheduling of multistage batch plants. Industrial \& Engineering Chemistry Research 44(24): 9175-9190.

Castro, P. M., I. Hariunkoski and I. E. Grossmann (2009). Optimal Short-Term Scheduling of Large-Scale Multistage Batch Plants. Industrial \& Engineering Chemistry Research 48(24): 11002-11016.

Dijkstra, E. W. (1959). A note on two problems in connexion with graphs. Numerische Mathematik 1(1): 269271.

Ferris, M. C., C. T. Maravelias and A. Sundaramoorthy (2009). Simultaneous Batching and Scheduling Using Dynamic Decomposition on a Grid. INFORMS Journal on Computing 21(3): 398-410.

Giannelos, N. F. and M. C. Georgiadis (2002). A simple new continuous-time formulation for short-term scheduling of multipurpose batch processes. Industrial \& Engineering Chemistry Research 41(9): 2178-2184.

Gimenez, D. M., G. P. Henning and C. T. Maravelias (2009a). A novel network-based continuous-time representation for process scheduling: Part I. Main concepts and mathematical formulation. Computers \& Chemical Engineering 33(9): 1511-1528.

Gimenez, D. M., G. P. Henning and C. T. Maravelias (2009b). A novel network-based continuous-time representation for process scheduling: Part II. General framework. Computers \& Chemical Engineering 33(10): 1644-1660.

Gupta, S. and I. A. Karimi (2003). An improved MILP formulation for scheduling multiproduct, multistage batch plants. Industrial \& Engineering Chemistry Research 42(11): 2365-2380.

Harjunkoski, I. and I. E. Grossmann (2002). Decomposition techniques for multistage scheduling problems using mixed-integer and constraint programming methods. Computers \& Chemical Engineering 26(11): 1533-1552.

Harjunkoski, I., C. T. Maravelias, P. Bongers, P. M. Castro, S. Engell, I. E. Grossmann, J. Hooker, C. Méndez, G. Sand and J. Wassick (2014). Scope for industrial applications of production scheduling models and solution methods. Computers \& Chemical Engineering 62(0): 161-193.

He, Y. H. and C. W. Hui (2006). Rule-evolutionary approach for single-stage multiproduct scheduling with parallel units. Industrial \& Engineering Chemistry Research 45(13): 4679-4692.

Ierapetritou, M. G. and C. A. Floudas (1998). Effective continuous-time formulation for short-term scheduling. 1. Multipurpose batch processes. Industrial \& Engineering Chemistry Research 37(11): 4341-4359.

Jain, V. and I. E. Grossmann (2001). Algorithms for hybrid MILP/CP models for a class of optimization problems. INFORMS Journal on Computing 13(4): 258-276.

Janak, S. L. and C. A. Floudas (2008). Improving unit-specific event based continuous-time approaches for batch processes: Integrality gap and task splitting. Computers \& Chemical Engineering 32(4-5): 913955.

Janak, S. L., C. A. Floudas, J. Kallrath and N. Vormbrock (2006). Production scheduling of a large-scale industrial batch plant. I. Short-term and medium-term scheduling. Industrial \& Engineering Chemistry Research 45(25): 8234-8252.

Kelly, J. D. and D. Zyngier (2008). Hierarchical decomposition heuristic for scheduling: Coordinated reasoning for decentralized and distributed decision-making problems. Computers \& Chemical Engineering 32(11): 2684-2705. 
Kondili, E., C. C. Pantelides and R. W. H. Sargent (1993). A General Algorithm for Short-Term Scheduling of Batch-Operations .1. Milp Formulation. Computers \& Chemical Engineering 17(2): 211-227.

Ku, H. M. and I. A. Karimi (1988). Scheduling in Serial Multiproduct Batch Processes with Finite Interstage Storage - A Mixed Integer Linear Program Formulation. Industrial \& Engineering Chemistry Research 27(10): 1840-1848.

Maravelias, C. T. (2006). A decomposition framework for the scheduling of single- and multi-stage processes. Computers \& Chemical Engineering 30(3): 407-420.

Maravelias, C. T. (2012a). General framework and modeling approach classification for chemical production scheduling. Aiche Journal 58(6): 1812-1828.

Maravelias, C. T. (2012b). On the combinatorial structure of discrete-time MIP formulations for chemical production scheduling. Computers \& Chemical Engineering 38: 204-212.

Maravelias, C. T. and I. E. Grossmann (2003). New general continuous-time state-task network formulation for short-term scheduling of multipurpose batch plants. Industrial \& Engineering Chemistry Research 42(13): 3056-3074.

Maravelias, C. T. and K. Papalamprou (2009). Polyhedral results for discrete-time production planning MIP formulations for continuous processes. Computers \& Chemical Engineering 33(11): 1890-1904.

Mauderli, A. and D. W. T. Rippin (1979). Production Planning and Scheduling for Multipurpose Batch Chemical-Plants. Computers \& Chemical Engineering 3(1-4): 199-206.

Mendez, C. A. and J. Cerda (2003). An MILP Continuous-Time Framework for Short-Term Scheduling of Multipurpose Batch Processes Under Different Operation Strategies. Optimization and Engineering 4(1-2): 7-22.

Mendez, C. A., J. Cerda, I. E. Grossmann, I. Harjunkoski and M. Fahl (2006). State-of-the-art review of optimization methods for short-term scheduling of batch processes. Computers \& Chemical Engineering 30(6-7): 913-946.

Mendez, C. A., G. P. Henning and J. Cerda (2000). Optimal scheduling of batch plants satisfying multiple product orders with different due-dates. Computers \& Chemical Engineering 24(9-10): 2223-2245.

Mendez, C. A., G. P. Henning and J. Cerda (2001). Short-term scheduling of multiproduct batch plants under limited resource capacity. Latin American Applied Research 31(5): 455-462.

Merchan, A. F., S. Velez and C. T. Maravelias (2013). Tightening methods for continuous-time mixed-integer programming models for chemical production scheduling. Aiche Journal 59(12): 4461-4467.

Mockus, L. and G. V. Reklaitis (1999). Continuous time representation approach to batch and continuous process scheduling. 1. MINLP formulation. Industrial \& Engineering Chemistry Research 38(1): 197203.

Nemhauser, G. L. and L. A. Wolsey (1988). Integer and combinatorial optimization. New York, Wiley.

Nie, Y. S., L. T. Biegler, C. M. Villa and J. M. Wassick (2014). Reactor Modeling and Recipe Optimization of RingOpening Polymerization: Block Copolymers. Industrial \& Engineering Chemistry Research 53(18): 7434-7446.

Pantelides, C. C. (1994). Unified Frameworks for Optimal Process Planning and Scheduling. 2nd Conference on Foundations of Computer Aided Process Operations, Snowmass, CO, CACHE Publications.

Papageorgiou, L. G. and C. C. Pantelides (1996). Optimal campaign planning scheduling of multipurpose batch semicontinuous plants .2. A mathematical decomposition approach. Industrial \& Engineering Chemistry Research 35(2): 510-529.

Pinto, J. M. and I. E. Grossmann (1995). A Continuous-Time Mixed-Integer Linear-Programming Model for Short-Term Scheduling of Multistage Batch Plants. Industrial \& Engineering Chemistry Research 34(9): 3037-3051.

Pinto, J. M. and I. E. Grossmann (1998). Assignment and sequencing models for the scheduling of process systems. Annals of Operations Research 81: 433-466.

Pochet, Y. and F. Warichet (2008). A tighter continuous time formulation for the cyclic scheduling of a mixed plant. Computers \& Chemical Engineering 32(11): 2723-2744.

Prasad, P. and C. T. Maravelias (2008). Batch selection, assignment and sequencing in multi-stage multiproduct processes. Computers \& Chemical Engineering 32(6): 1106-1119.

Reklaitis, G. V., Ed. (1978). Review of Scheduling of Process Operation. AIChE Symp. Ser.

Roe, B., L. G. Papageorgiou and N. Shah (2005). A hybrid MILP/CLP algorithm for multipurpose batch process scheduling. Computers \& Chemical Engineering 29(6): 1277-1291. 
Schilling, G. and C. C. Pantelides (1996). A simple continuous-time process scheduling formulation and a novel solution algorithm. Computers \& Chemical Engineering 20: S1221-S1226.

Shah, N., C. C. Pantelides and R. W. H. Sargent (1993). A General Algorithm for Short-Term Scheduling of Batch-Operations .2. Computational Issues. Computers \& Chemical Engineering 17(2): 229-244.

Subrahmanyam, S., G. K. Kudva, H. H. Bassett and J. F. Pekny (1996). Application of distributed computing to batch plant design and scheduling. Aiche Journal 42(6): 1648-1661.

Sundaramoorthy, A. and I. A. Karimi (2005). A simpler better slot-based continuous-time formulation for short-term scheduling in multipurpose batch plants. Chemical Engineering Science 60(10): 26792702.

Sundaramoorthy, A. and C. T. Maravelias (2008a). Modeling of storage in batching and scheduling of multistage processes. Industrial \& Engineering Chemistry Research 47(17): 6648-6660.

Sundaramoorthy, A. and C. T. Maravelias (2008b). Simultaneous batching and scheduling in multistage multiproduct processes. Industrial \& Engineering Chemistry Research 47(5): 1546-1555.

Sundaramoorthy, A., C. T. Maravelias and P. Prasad (2009). Scheduling of Multistage Batch Processes under Utility Constraints. Industrial \& Engineering Chemistry Research 48(13): 6050-6058.

Susarla, N., J. Li and I. A. Karimi (2010). A novel approach to scheduling multipurpose batch plants using unitslots. Aiche Journal 56(7): 1859-1879.

Velez, S. and C. T. Maravelias (2013a). A branch-and-bound algorithm for the solution of chemical production scheduling MIP models using parallel computing. Computers \& Chemical Engineering 55(0): 28-39.

Velez, S. and C. T. Maravelias (2013b). Mixed-Integer Programming Model and Tightening Methods for Scheduling in General Chemical Production Environments. Industrial \& Engineering Chemistry Research 52(9): 3407-3423.

Velez, S. and C. T. Maravelias (2013c). Reformulations and Branching Methods for Mixed-Integer Programming Chemical Production Scheduling Models. Industrial \& Engineering Chemistry Research 52(10): 3832-3841.

Velez, S. and C. T. Maravelias (2015). Theoretical framework for formulating MIP scheduling models with multiple and non-uniform discrete-time grids. Computers \& Chemical Engineering 72(0): 233-254.

Velez, S., A. Sundaramoorthy and C. T. Maravelias (2013). Valid Inequalities Based on Demand Propagation for Chemical Production Scheduling MIP Models. Aiche Journal 59(3): 872-887.

Zhang, X. and R. W. H. Sargent (1996). The optimal operation of mixed production facilities - A general formulation and some approaches for the solution. Computers \& Chemical Engineering 20(6-7): 897904. 